See discussions, stats, and author profiles for this publication at: https://www.researchgate.net/publication/234013313

\title{
Media and Intima Thickness and Texture Analysis of the Common Carotid Artery
}

Chapter·April 2012

CITATIONS

0

2 authors:

Christos P Loizou

University of Cyprus

156 PUBLICATIONS 1,847 CITATIONS

SEE PROFILE
READS

26

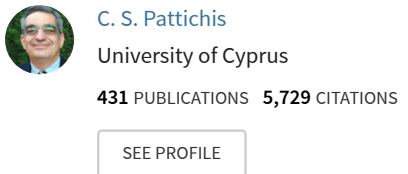

Some of the authors of this publication are also working on these related projects:

GRANATUM "A social collaborative working space semantically interlinking biomedical researchers knowledge and data for the design and execution of in-silico models and experiments in cancer chemoprevention" FP7 Project View project

RSNA 2017 View project 


\title{
Media and Intima Thickness and Texture Analysis of the Common Carotid Artery
}

\author{
C.P. Loizou, M. Pantzaris, and C.S. Pattichis
}

\begin{abstract}
The intima-media thickness (IMT) of the common carotid artery (CCA) 4 is widely used as an early indicator for the development of cardiovascular disease 5 (CVD). It was proposed but not thoroughly investigated that the media layer (ML) 6 thickness (MLT), its composition, and texture may be indicative of cardiovascular 7 risk and for differentiating between patients with high and low risk. In this study, 8 we investigate an automated snakes segmentation method for segmenting the ML 9 and the intima layer (IL) and measurement of the MLT and the intima layer 10 thickness (ILT) in ultrasound images of the CCA. We furthermore investigate 11 the application of texture analysis of the ML of the CCA and how texture is 12 affected by age and gender. The snakes segmentation method was used, and was 13 evaluated on 100 longitudinal ultrasound images acquired from asymptomatic 14 subjects, against manual segmentation performed by a neurovascular expert. The 15 mean \pm standard deviation (sd) for the first and second sets of manual and 16 the automated IMT, MLT, and ILT measurements were $0.71 \pm 0.17 \mathrm{~mm}, 0.72 \pm 17$ $0.17 \mathrm{~mm}, 0.67 \pm 0.12 \mathrm{~mm}, 0.25 \pm 0.12 \mathrm{~mm}, 0.27 \pm 0.14$, and $0.25 \pm 0.11 \mathrm{~mm}$; and 18 $0.43 \pm 0.10 \mathrm{~mm}, 0.44 \pm 0.13 \mathrm{~mm}$, and $0.42 \pm 0.10 \mathrm{~mm}$, respectively. There was 19 overall no significant difference between the manual and the automated IMC, ML, 20 and IL segmentation measurements. Therefore, the automated segmentation method 21 proposed in this study may be used successfully in the measurement of the MLT and 22 ILT complementing the manual measurements. MLT was also shown to increase 23 with age (for both the manual and the automated measurements). Following the 24
\end{abstract}

C.P. Loizou $(\square)$

Department of Computer Science, School of Sciences, Intercollege,

AQ1 92 Ayias Phylaxeos Str., P.O. Box 51604, CY-3507, Limassol, Cyprus

e-mail: loizou.christos@ucy.ac.cy; loizou.c@lim.intercollege.ac.cy

M. Pantzaris

Cyprus Institute of Neurology and Genetics, Nicosia, Cyprus

C.S. Pattichis

Department of Computer Science, University of Cyprus, Nicosia, Cyprus 
segmentation of the three structures, we also investigated the application of texture 25 analysis of the ML of the CCA and how texture is affected by age and gender. 26 The 100 images were separated into three different age groups, namely below 50, 27 between 50 and 60 , and above 60 years old. Furthermore, the images were separated 28 according to gender. A total of 61 different texture features were extracted from the 29 intima layer (IL), the ML, and the intima-media complex (IMC). We have found 30 that male patients tended to have larger media layer thickness (MLT) values as 31 compared to the MLT of female patients of the same age. We have also found 32 significant differences among texture features extracted from the IL, ML, and IMC 33 from different age groups. Furthermore, for some texture features, we found that 34 they follow trends that correlate with a patient's age. For example, the gray-scale 35 median GSM of the ML falls linearly with increasing MLT and with increasing age. 36 Our findings suggest that ultrasound image texture analysis of the media layer has 37 potential as an assessment biomarker for the risk of stroke.

Keywords Snakes - Ultrasound imaging - Media layer - Texture analysis 39

- Common carotid artery

\section{Introduction}

Cardiovascular disease (CVD - coronary artery disease, cerebrovascular disease, 42 and peripheral artery disease) is the third leading cause of death and adult disability 43 in the industrial world after heart attack and cancer (as reported by the World Health 44 Organization). According to [1], 80 million American adults have one or more types 45 of CVD of whom about half are estimated to be of age 65 or older. Of all the 46 deaths caused by CVD among adults aged 20 and older, an estimated 13 millions 47 are attributed to coronary heart disease and to stroke, with atherosclerosis as the 48 underlying cause. A recent study by the World Health Organization estimates that 49 by 2015, 20 million people will die from cerebrovascular disease (mainly from heart 50 attack and stroke).

Atherosclerosis causes thickening of the artery walls and the intima-media 52 thickness (IMT) (see Fig. 1a) is used as a validated measure for the assessment of 53 atherosclerosis [2]. Specifically, an increased IMT is correlated with an augmented 54 risk of brain infarction or cardiac attack [3]. Moreover, the presence of carotid 55 plaques has been correlated not only to CVD but also to degenerative pathologies 56 such as vascular dementia and Alzheimer's disease [4]. Hence the assessment of 57 carotid wall status is also essential for early identification of risk conditions in 58 asymptomatic patients. Traditionally, the IMT is measured by manual delineation 59 of the intima and the adventitia layers [2] (see Fig. 1a, interface I5 and I7). Manual 60 tracing of the lumen diameter (see Fig. 1a, band Z4) and the IMT by human experts 61 requires substantial experience; it is time consuming, and varies according to the 62 training, experience, and the subjective judgment of the experts. The manual mea- 63 surements suffer, therefore, from considerable inter- and intra-observer variability 64 [2-10]. It should be noted that parts of this work were also published in $[11,12]$. 


\section{Editor's Proof}
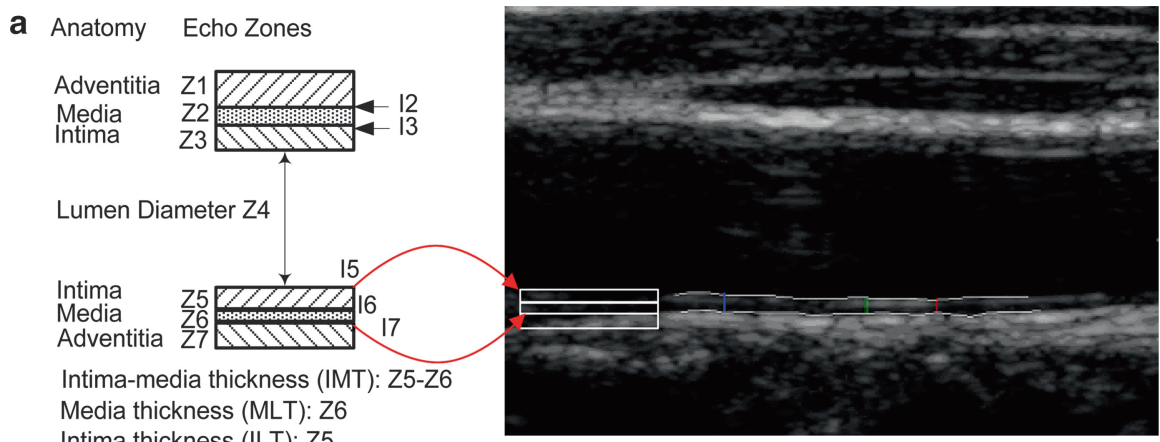

Intima thickness (ILT): Z5

b

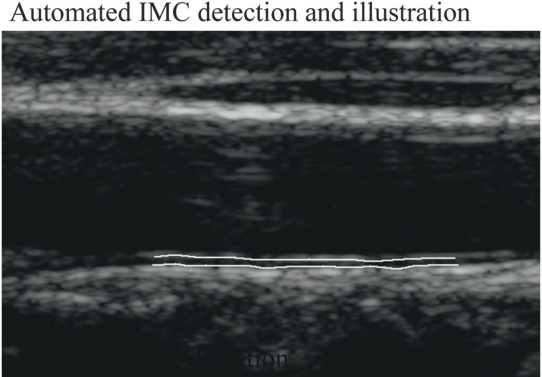

e
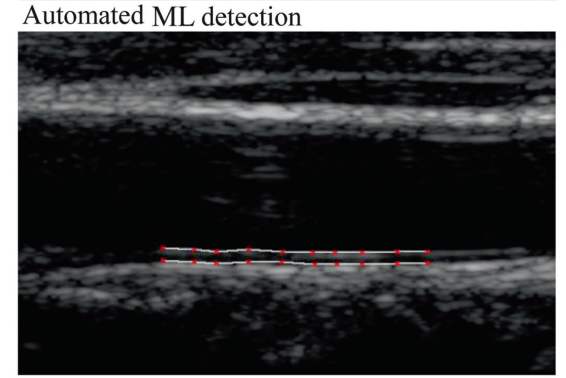

Manual IMC delineation

g

IMC (Manual)

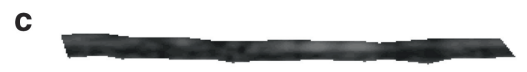

IMC Automated

d

ML (Automated)

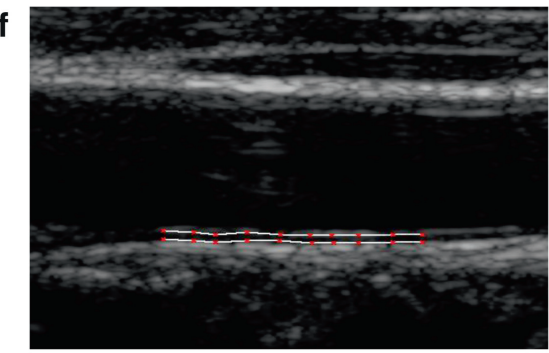

Manual ML delineation

h

ML (Manual)

Fig. 1 (a) Illustration of the intima-media complex (IMC, bands Z5 and Z6) of the far wall of the common carotid artery and the automatic IMC segmentation [10]. The media layer thickness (MLT) is defined as the distance between the intima-media and the media-adventitia interface (band Z6), (b) automated ML detection, (c) automated extracted IMC band, (d) automated extracted ML, (e) manual IMC delineation, (f) manual ML delineation, (g) manual extracted IMC, and (h) manual extracted ML. Source [12], (c) IEEE 2009

In the last 20 years, several automated techniques for the segmentation and mea- 66 surement of the IMT from longitudinal ultrasound images of the common carotid 67 artery (CCA) have been developed [9-14]. However, there are no studies published 68 in the literature reporting both the manual and the automated segmentation and 69 
measurement of the media layer (ML) and the intima layer (IL) of the CCA in 70 ultrasound imaging. There are only three studies in the literature where manual 71 measurements of the MLT were reported [8, 15, 16]. Earlier research showed that 72 the media layer thickness (MLT) in peripheral arteries does not change significantly 73 with age and that it ranges from 125 to $350 \mu \mathrm{m}$ [8]. In [15], manual measurements 74 of the thickness of the CCA IMT and MLT were carried out by an expert on 10075 subjects aged 70 years old. In this study [15], it was shown that subjects with CVD 76 (coronary heart disease, myocardial infarction, or stroke) had a significantly thinner 77 ML and a thicker IL than healthy subjects. Furthermore, in [16], the IMT, MLT, and 78 ILT of 90 healthy subjects (aged between 10 and 90 years) were manually measured 79 at their radial and anterior tibial arteries. It was shown that age was strongly related 80 with IMT, MLT, and ILT for both peripheral arteries.

The objective of this study was to develop and evaluate a snakes segmentation 82 system enabling the automated segmentation and measurement of the ML and 83 IL in ultrasound images of the CCA and investigate their variability with age 84 groups. We also propose to study changes in textural characteristics that can be 85 associated with disease progression for different age groups. Here, we note that 86 for fully developed plaques in the CCA, texture features derived from statistical, 87 model based, and Fourier based methods have been used to characterize and 88 classify carotid atheromatous plaques from B-mode ultrasound images $[11,30]$. 89 We, furthermore, investigated whether textural characteristics extracted from the 90 IL, the ML, and the IMC of the CCA, segmented manually by an expert and 91 automatically by a snakes segmentation system [10,12], can be associated with the 92 increase in age gender or MLT. Ultimately, texture feature characteristics that vary 93 with age, gender, or MLT might be used to asses the risk of stroke.

The ML (see Fig. 1a, band Z6) is ultrasonographically characterized by an 95 echolucent region, predominantly composed of smooth muscle cells of the media 96 band of the arterial wall and probably by the extracellular matrix of the intima band, 97 which cannot be distinguished from the smooth muscle cell with ultrasound [17]. 98 Due to the acoustic impedance mismatches, the arterial wall bands (intima- 99 media-adventitia) can produce typical bright-dark-bright patterns on ultrasound 100 images [2]. It is, furthermore, proposed but not thoroughly investigated, that not 101 only the IMT but rather the ML (its composition and thickness) may be used for 102 evaluating the risk of a patient to develop a stroke and accounts in general for the 103 risk of the CVD by differentiating between patients with high and low risk. 104

The IL is a thin layer, the thickness of which increases with age, from a single 105 cell layer at birth to $250 \mu \mathrm{m}$ at the age of 40 for nondiseased individuals [5]. Further, 106 adaptive physiological thickening of the IL occurs at places where the wall tension 107 is increased, such as arterial bifurcations and on the ML of the artery and may be 108 either eccentric or diffuse [5]. Furthermore, the intima-media complex (IMC) (see 109 also Fig. 1a) becomes more difficult to detect as the age of patients increases, due 110 to the presence of acoustic holes (echo-dropouts), in the IL [6]. The intimal band 111 (see band Z5 in Fig. 1a) may appear as a thin low-contrast structure and, therefore, 112 it is difficult to reliably draw boundaries because smoothing can move the structure 113 edges or make them disappear $[2,7]$. 
Earlier research [8] showed that the media layer thickness (MLT) does not change 115 significantly with age $(125 \mu \mathrm{m}<\mathrm{MLT}<350 \mu \mathrm{m})$. In a recent study by our group, 116 the median (IQR) of intima layer thickness (ILT), MLT, and IMT were computed 117 from 100 ultrasound images of 42 female and 58 male asymptomatic subjects aged 118 between 26 and 95 years old, with a mean age of 54 years to be as follows: $0.43 \mathrm{~mm} 119$ (0.12), $0.23 \mathrm{~mm}(0.18)$, and $0.66 \mathrm{~mm}(0.18)$, respectively [12].

In [33], a method has been presented for quantifying the reflectivity of the ML 121 of the distal CCA. It was shown that the GSM of the IM layer is the earliest 122 change representing atherosclerotic disease in the arterial wall that can currently 123 be imaged in vivo. This may be the first marker of atherosclerosis and may 124 precede the development of a significant increase in IMT. This would enable earlier 125 identification of high-risk individuals based on the analysis of the CCA artery wall 126 textural characteristics. In [34], the early structural changes of the CCA in familial 127 hypercholesterolemia were investigated. It was shown that textural characteristics 128 extracted from the IMC were significantly different between patients with and 129 without hypercholesterolemia. In [35], the authors reported on the properties of the 130 GSM of the IMC from a random sample of 1,016 subjects aged exactly 70. They 131 found that the GSM of the IMC of the CCA is closely related to the echogenecity in 132 overt carotid plaques.

While there are several earlier studies suggesting that the instability of the carotid 134 atheromatous plaques can be characterized from B-mode ultrasound images [6,30], 135 we have not found any other studies reported in the literature where the ML textural 136 characteristics have been shown to be associated with the risk of stroke. While in 137 $[6,30]$, the echogenecity in atherosclerotic carotid plaques was evaluated through 138 the gray-scale median (GSM), there are very few attempts made to characterize the 139 IL, the ML, and the intima-media complex (IMC) with a similar gray-scale image 140 intensity analysis. It is evident from the visual inspections of the IMC in the CCA 141 that a great variation in echogenecity does exist. However, the usefulness of this 142 information has not yet been studied.

In [30], the morphology of atherosclerotic carotid plaque was investigated based 144 on the textural characteristic extracted from 230 ultrasound images of carotid 145 plaque, where it was shown that it is possible to identify a group of patients, 146 symptomatic or asymptomatic, at risk of stroke based on these texture features. 147 It was further documented in [36], that carotid endarterectomy in asymptomatic 148 individuals with stenosis greater than $70 \%$ reduces the risk of stroke from $2 \% 149$ per year to $1 \%$ per year. In another study [37], the relationship of the IMT in 150 the CCA and atherosclerosis was investigated on 182 symptomatic patients (mean 151 age 67 years). It was shown that the IMT was correlated to age, male gender, 152 ischemic heart disease, and presence of plaque or stenosis in any of the carotid 153 bifurcations. In a recent study [38], where the alterations of the CCA with age in 154 ultrasound images were investigated, it was shown that the diastolic and systolic 155 lumen diameters are increasing with age. This reduces wall stress as the elasticity 156 of the wall decreases with age. 


\section{Materials and Methods}

\subsection{Recording of Ultrasound Images}

A total of $100 \mathrm{~B}$-mode longitudinal ultrasound images of the CCA used for the 160 IMC, ML, and IL segmentations were recorded using the ATL HDI-3000 ultrasound 161 scanner (Advanced Technology Laboratories, Seattle, USA), with a linear probe 162 (L74), with a recording frequency of $7 \mathrm{MHz}$, a velocity of $1,550 \mathrm{~m} / \mathrm{s}$ and 1 cycle 163 per pulse, which resulted to a wavelength (spatial pulse length) of $0.22 \mathrm{~mm}$ and an 164 axial system resolution of $0.11 \mathrm{~mm}$. The technical characteristics of the ultrasound 165 scanner (multielement ultrasound scan head, operating frequency, acoustic aperture, 166 and transmission focal range) have already been published in [10]. Digital images 167 were resized using the bicubic method to a standard pixel density of 16.66 pixels per 168 $\mathrm{mm}$ with a resolution of $0.06 \mathrm{~mm}$. This was carried out due to the small variations in 169 the number of pixels per mm of image depth (i.e., for deeply situated carotid arteries, 170 image depth was increased and, therefore, digital image spatial resolution would 171 have decreased) and in order to maintain uniformity in the digital image spatial 172 resolution [19]. The images were logarithmically compressed and were recorded 173 digitally on a magneto-optical drive at size of $768 \times 576$ pixels with 256 gray 174 levels. The images were recorded at the Cyprus Institute of Neurology and Genetics, 175 Nicosia, Cyprus, from 42 female and 58 male asymptomatic subjects aged between 176 26 and 95 years old, with a mean age of 54 years. The images were separated into 177 three different age groups depending on age, namely, below 50, between 50 and 60, 178 and above 60 years old, with 27,36, and 37 subjects in each group, respectively, 179 and also separated into 58 male and 42 female subjects. These subjects had not 180 developed clinical symptoms, such as a stroke or a transient ischemic attack (TIA). 181

\subsection{Image Normalization}

Brightness adjustments of ultrasound images were carried out in this study based on 183 the method introduced in [20] and also used in [10]. It was shown that this method 184 improves image compatibility by reducing the variability introduced by different 185 gain settings, different operators, different equipment, and facilitates ultrasound 186 tissue comparability. Algebraic (linear) scaling of the image was performed by 187 linearly adjusting the image so that the median gray level value of the blood was 188 $0-5$, and the median gray level of the adventitia (artery wall) was 180-190 [20]. 189 The scale of the gray level of the images ranged from 0 to 255 . Thus the brightness 190 of all pixels in the image was readjusted according to the linear scale defined by 191 selecting the two reference regions. It is noted that a key point to maintaining a 192 high reproducibility was to ensure that the ultrasound beam was at right angles 193 to the adventitia, adventitia was visible adjacent to the plaque, and that for image 194 normalization a standard sample consisting of the half of the width of the brightest 195 area of adventitia was obtained. 


\subsection{Manual Measurements}

A neurovascular expert delineated manually (using the mouse) the IMC [10], the 198 ML, and the IL on 100 longitudinal ultrasound images of the CCA after image 199 normalization. The IMC was measured by selecting $20-40$ consecutive points for 200 the intima (see Fig. 1a, interface I5) and the adventitia (see Fig. 1a, interface I7) 201 layers, and the ML (see Fig. 1a, band Z6) by selecting 10-20 consecutive points for 202 the media (see Fig. 1, interface I6) and the adventitia layers at the far wall of the 203 CCA. The IL (see Fig. 1a, band Z5) was then derived by subtracting the ML from 204 the IMC. The manual delineations were performed using a system implemented in 205 MATLAB ${ }^{\circledR}$ from our group. The measurements were performed between $1 \mathrm{~cm}$ and 206 $2 \mathrm{~cm}$ proximal to the bifurcation of the CCA on the far wall [10] over a distance of 207 $1.5 \mathrm{~cm}$ starting at a point $0.5 \mathrm{~cm}$ and ending at a point $2.0 \mathrm{~cm}$ proximal to the carotid 208 bifurcation. The bifurcation of the CCA was used as a guide and all measurements 209 were made from that region. The IMT, the MLT, and the ILT were then calculated 210 as the average of all the measurements. The measuring points and delineations 211 were saved for comparison with the snakes segmentation method. Two sets of 212 measurements were carried out by the neurovascular expert with a 6-month interval. 213 All sets of manual segmentation measurements were performed by the expert in 214 a blinded manner, both with respect to identifying the subject and to the image 215 delineation.

\subsection{IMC, ML, and IL Snakes Segmentation}

A total of 100 ultrasound images of the CCA were segmented using the automated 218 segmentation system presented in [12], to identify IMC, ML, and IL. Segmentation 219 was carried out after image normalization using the automated snakes segmentation 220 system proposed and evaluated on ultrasound images of the CCA in [10], which 221 is based on the Williams and Shah [21]. Initially the IMC was segmented by a 222 snake segmentation system as proposed in [21], where the boundaries I5 (lumen- 223 intima interface) and I7 (media-adventitia interface) were extracted. Details about 224 the implementation of the algorithm can be found in [10].

The upper side of the ML (see Fig. 1, Z6) was estimated by deforming the lumen- 226 intima interface (boundary I5) by $0.36 \mathrm{~mm}$ ( 6 pixels) downwards and then deformed 227 by the snakes segmentation algorithm proposed in [10] in order to fit to the media 228 boundary (see Fig. 1a, interface I6). This displacement of $0.36 \mathrm{~mm}$ is based on the 229 observation that the manual mean IMT (IMT mean) is $0.71 \mathrm{~mm}$ (12 pixels), and lies 230 between $0.54 \mathrm{~mm}$ (minimum or 9 pixels) and $0.88 \mathrm{~mm}$ (maximum or 15 pixels) [10]. 231 Therefore, the displacement of the contour, in order to estimate the media should be 232 in average $0.36 \mathrm{~mm}$ ( 6 pixels times $0.06 \mathrm{~mm}$ ) downwards, which is half of the size of 233 the IMT (the distance between I5 and I7, where I7 is the media-adventitia interface). ${ }^{234}$ 
In order to achieve standardization in extracting the thickness of IMC, ML, 235 and IL, segments with similar dimensions were divided based on the following 236 procedure. A region of interest of $9.6 \mathrm{~mm}$ (160 pixels) in length, located 1-2 cm 237 proximal to the bifurcation of the CCA on the far wall, was extracted. This was 238 done by estimating the center of the IMC area and then selecting $4.8 \mathrm{~mm}$ ( 80 pixels) 239 left and $4.8 \mathrm{~mm}$ (80 pixels) right of the center of the segmented IMC. The selection 240 of the same ML area in length from each image is important in order to be able to 241 make comparable measurements between images and patient groups.

The novelty of the proposed methodology lies in the algorithmic integrated 243 approach that facilitates the automated segmentation and measurements of IL 244 and ML.

\subsection{Texture Analysis}

In order to estimate textural characteristics extracted from the IL, ML, and the 247 IMC, a total of 61 different texture features were extracted both from the manual 248 and the automated segmented regions of interest where only the most significant 249 are presented. The following texture feature set algorithms were used: (i) statistical 250 features [11,12]: (a) mean, (b) variance, (c) median value, (d) skewness, (e) kurtosis, 251 (f) energy, and (g) entropy. (ii) Spatial gray level dependence matrices (SGLDM) 252 as proposed by Haralick et al. [39]: (a) angular second moment, (b) contrast, (c) 253 correlation, (d) sum of squares variance, (e) inverse difference moment, (f) sum 254 average, (g) sum variance, (h) sum entropy, (i) entropy, (j) difference variance, 255 (k) difference entropy, and (1) information measures of correlation. For a chosen 256 distance $d$ (in this work $d=1$ was used) and for angles $\theta=00,450,900$, and 257 1,350 , we computed four values for each of the above texture measures. (iii) Gray 258 level difference statistics (GLDS) [40]: (a) homogeneity, (b) contrast, (c) energy, (d) 259 entropy, and (e) mean. The above features were calculated for displacements $\delta=260$ $(0,1),(1,1),(1,0),(1,-1)$, where $\delta \equiv(\Delta x, \Delta y)$, and their mean values were taken. 261 (iv) Neighborhood gray tone difference matrix (NGTDM) [41]: (a) coarseness, (b) 262 contrast, (c) busyness, (d) complexity, and (e) strength. (v) Statistical feature matrix 263 (SFM) [42]: (a) coarseness, (b) contrast, (c) periodicity, and (d) roughness. (vi) 264 Laws texture energy measures (LTEM) [42]: LL-texture energy from LL-kernel, 265 EE-texture energy from EE-kernel, SS-texture energy from SS-kernel, LE-average 266 texture energy from LE- and EL-kernels, ES-average texture energy from ES- and 267 SE-kernels, and LS-average texture energy from LS- and SL-kernels. (vii) Fractal 268 dimension texture analysis (FDTA) [42]: The Hurst coefficients for dimensions four, 269 three, and two were computed. (viii) Fourier power spectrum (FPS) [42]: (a) radial 270 sum and (b) angular sum. 


\subsection{Statistical Analysis}

We computed the mean and median values for the IMT (IMT $\left.\mathrm{IMean}_{\text {, IMT }} \mathrm{IMedian}\right), 273$ the MLT (MLT mean $_{\text {MLT }}$ median $)$, and the ILT (ILT mean $_{\text {ILT }}$ ILdian $_{\text {med }}$, the inter- 274 observer error for the IMT, MLT, and the ILT $(\mathrm{se}=\sigma \sqrt{2})$ [9] as well as the IMT 275 ratio [15] $\left(\mathrm{IMT}_{\text {ratio }}=\mathrm{MLT} / \mathrm{ILT}\right)$. We also calculated the coefficient of variation, 276 CV\%, for the IMT, MLT, and ILT, respectively, which describes the difference 277 as a percentage of the pooled mean values, where for the media $\mathrm{CV} \%_{\text {media }}=278$ $\left(\mathrm{se}_{\text {media }} \times 100\right) / \mathrm{MLT}_{\text {media }}$ [9]. The above measurements were also computed for the 279 three different age groups, namely, below 50, between 50 and 60, and above 60 years 280 old and for the male and female subjects. Additionally, in order to assess the intra- 281 observer variability for the neurovascular expert, the manual MLT measurements 282 were repeated 6 months after the first set of measurements.

The Wilcoxon rank sum test, which calculates the difference between the sum 284 of the ranks of two dependent samples, was also used in order to identify if a 285 significant difference (S) or not (NS) exists at $p<0.05$, between the manual and 286 the snakes segmentation measurements, of IMC, ML, IL, and for the $\mathrm{IMT}_{\text {ratio }}$ for all 287 100 image, and between the manual and the automated segmentation measurements 288 IMC, ML, and IL for the three different age groups (below 50, between 50 and 289 60 , and above 60 years old). Furthermore, the Mann-Whitney rank sum test was 290 also applied in order to identify differences between male and female subjects. 291 Also the correlation coefficient, $\rho$, between the manual and the automated IMT, 292 MLT, and ILT measurements, was investigated, which reflects the extent of a linear 293 relationship between two data sets [22].

In order to investigate how the automated media snakes segmentation method 295 differs from the manual delineation results, we have investigated the regression 296 lines for the mean values of the MLT vs. age. Regression analysis was also used 297 in order to demonstrate the relationship between the IMT, MLT, and ILT mean 298 manual measurements and age of the subjects. Bland-Altman plots [23], with $95 \% 299$ confidence intervals, were also used to further evaluate the agreement between the 300 manual and the automated media segmentation measurements. The results of this 301 analysis are, however, not presented in this study as are the expansion of the studies 302 are published in [10-12]. Furthermore, box plots for the manual and the automated 303 IMT, MLT, and ILT mean measurements were plotted, as well as for the manual 304 IMT, MLT, and ILT measurements for the three different age groups (below 50, 305 between 50 and 60, and above 60 years old).

\section{Results}

Figure 1 illustrates an original normalized ultrasound image of the carotid artery 308 with the automated segmentation of the IMC and ML in (a) and (b), the extracted 309 automated IMC and ML bands in (c) and (d), the manual delineations of the IMC 310 and ML in (e) and (f), and the extracted manual IMC and ML bands in (g) and (h), 311 
respectively. It should be noted that the size of the IMC and ML areas presented in 312 Fig. 1c, d, g, h is larger than its original size (enlarged to $300 \times 20$ pixels in order to 313 be better visualized) and does not represent the original truth size (see also Sect. 2.4). 314

The manual (first and second set of measurements) and the automated mean and 315 median measurements for all 100 images, and for the three different age groups, of 316 the IMC, ML, and IL, are presented in Table 1, as well as the inter-observer error, se, 317

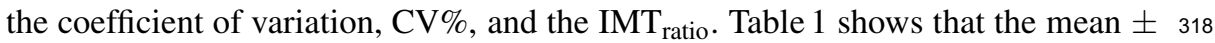
sd for the manual first set and the automated IMT measurements at time zero were 319 $0.71 \pm 0.17 \mathrm{~mm}$ and $0.67 \pm 0.12 \mathrm{~mm}$, for the manual first set and the automated MLT 320 measurements were $0.25 \pm 0.12 \mathrm{~mm}$ and $0.25 \pm 0.11 \mathrm{~mm}$, and for the manual first 321 set and the automated ILT measurements were $0.43 \pm 0.10 \mathrm{~mm}$ and $0.42 \pm 0.13 \mathrm{~mm}, \quad 322$ respectively. The inter-observer errors, se, for the manual first set and the automated 323 IMT segmentation measurements were 0.11 and 0.08 , whereas for the MLT and ILT 324 measurements were 0.09 and 0.08 , and 0.07 and 0.07 , respectively. The coefficients 325 of variation, $\mathrm{CV} \%$, for the manual first set and the automated IMT measurements 326 were $17.3 \%$ and $12.6 \%$, whereas for the manual first set and the automated MLT 327 and ILT segmentation measurements, were $34 \%$ and $12.3 \%$, and $16.7 \%$ and $11.7 \%$, 328 respectively. The $\mathrm{IMT}_{\text {ratio }}$, for the first set of manual measurements for all 100329 images and for the three different age groups (below 50, between 50 and 60, 330 and above 60 years old) were $0.5834 \pm 0.323 \mathrm{~mm}, 0.4314 \pm 0.242 \mathrm{~mm}, 0.634 \pm 331$ $0.273 \mathrm{~mm}, 0.6867 \pm 0.273 \mathrm{~mm}$, respectively. Similar values, but slightly higher, 332 were observed for the second set of manual measurements carried out at month 6 , for 333 the IMT, MLT, and ILT (see Table 1). For the IMT $_{\text {ratio }}$ using the Wilcoxon rank sum 334 test, there was no significance difference between the automated and the second set 335 of manual measurements ( $p=0.698$ ); however, there was a significance difference 336 between the automated and the first set of manual measurements $(p=0.031)$ and 337 between the first and the second set of manual measurements $(p=0.011)$.

Table 2 presents the results of the Mann-Whitney rank sum test performed 339 between the male and the female subjects for all three segmentation measurements 340

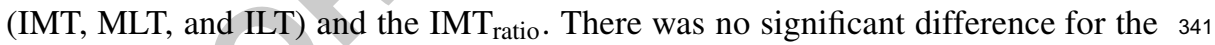
MLT between the male and the female subjects for the first set and automated 342 measurements. Significant differences between the male and the female subjects 343 were only found for the second set of manual measurements for the IMT $(p=0.01) 344$ and ILT $(p=0.013)$. There was also no significant difference for the $\mathrm{IMT}_{\text {ratio }} 345$ between the male and the female subjects.

The Wilcoxon rank sum test between the manual and the automated IMT, MLT, 347 ILT, and IMT $\mathrm{T}_{\text {ratio }}$ measurements was also performed between the three different age 348 groups and it is shown in Table 3. The Wilcoxon rank sum test and the correlation 349 coefficient (with 95\% confidence) results performed between the manual and the 350

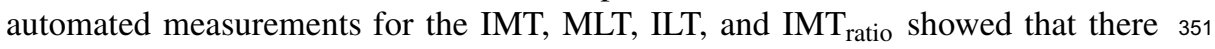
were no significant differences between these sets of measurements.

The automated MLT values (MLT_A) vs. age using regression analysis gave 353 an equation of MLT $A=0.029+0.0041 \times$ AGE, where the standard error for 354 intercept and slope were 0.0661 and 0.001 , respectively. The mean square error 355 due to regression was 0.1509 , the residual mean square was 0.013 resulting in an 356 


\section{Editor's Proof}

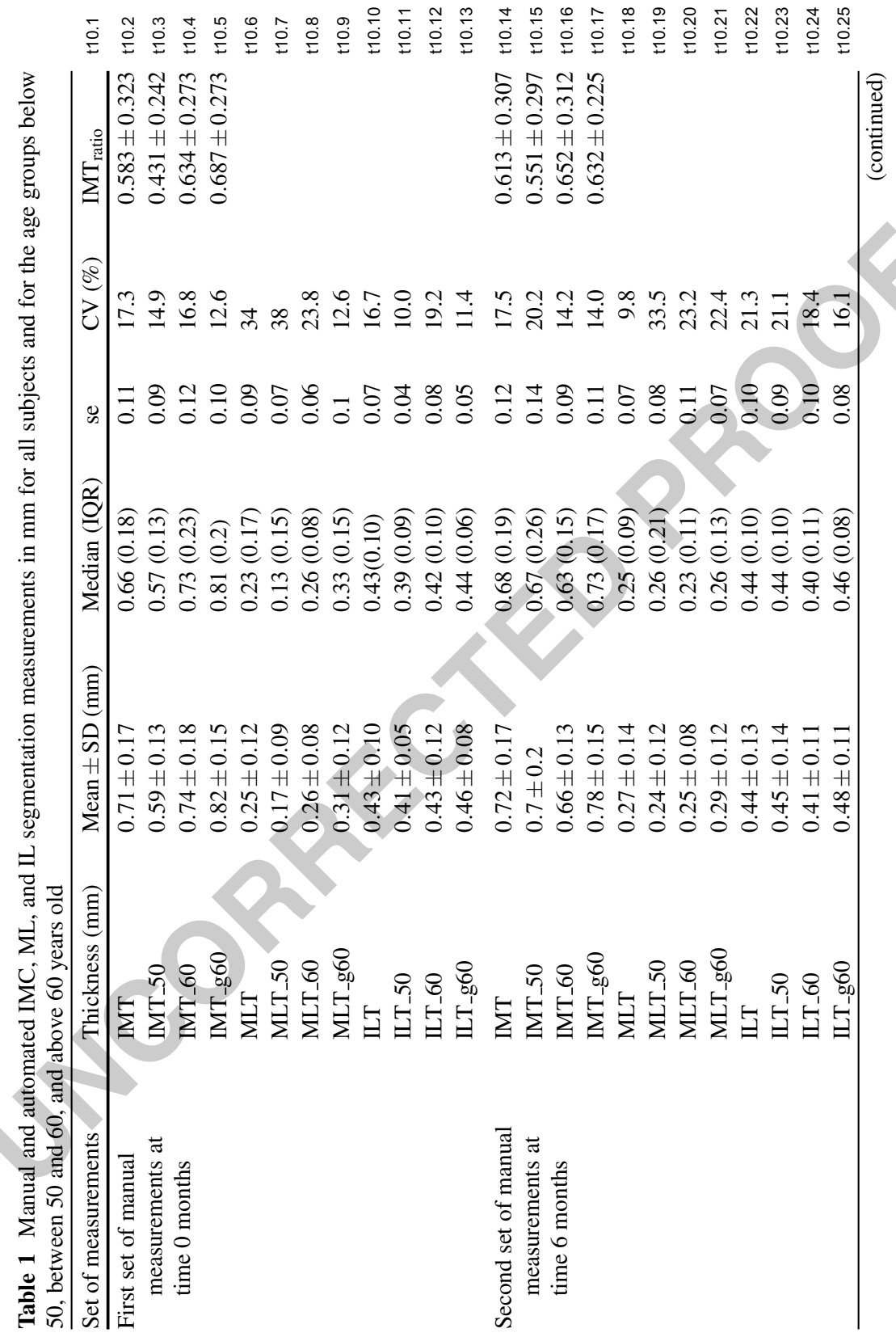




\section{Editor's Proof}

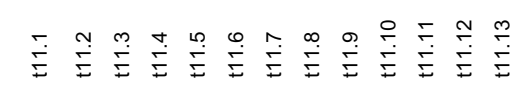

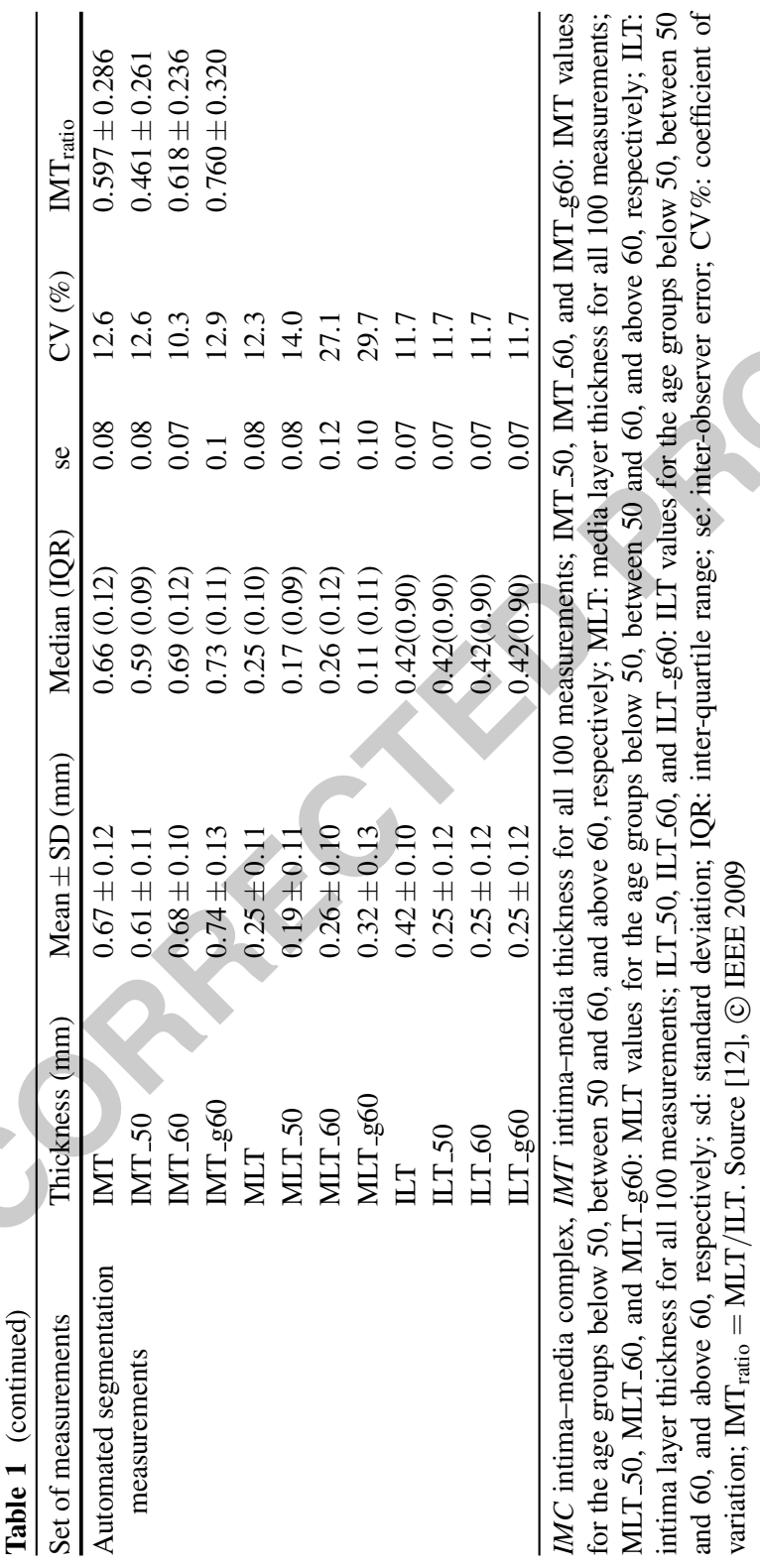




\section{Editor's Proof}

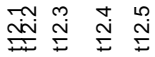

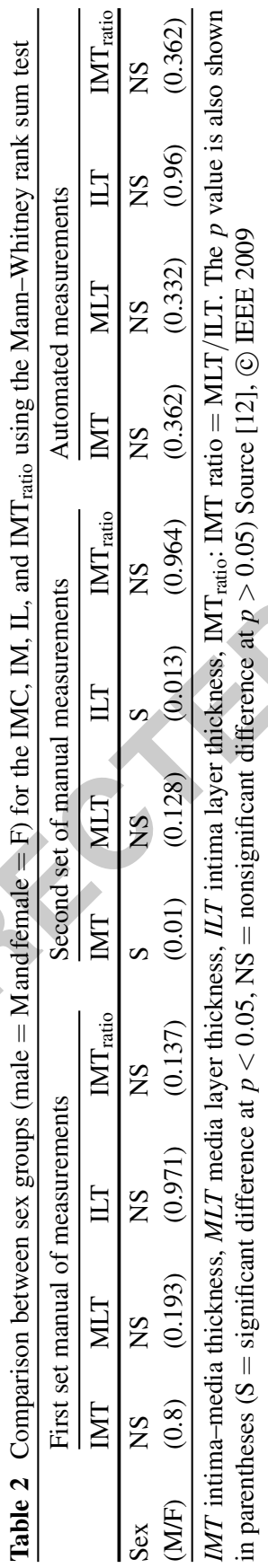




\section{Editor's Proof}

110

C.P. Loizou et al.

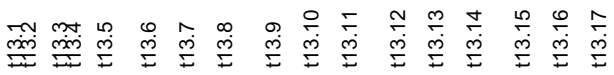

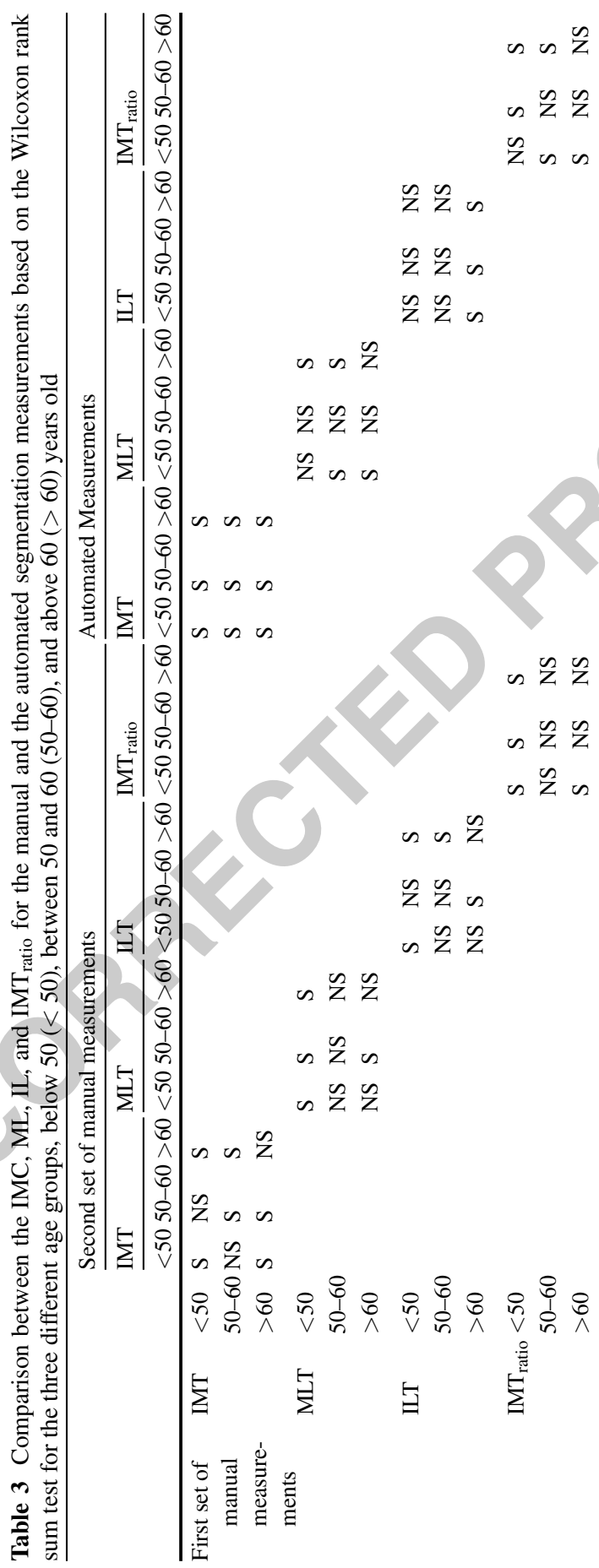




\section{Editor's Proof}

Media and Intima Thickness and Texture Analysis of the Common Carotid Artery

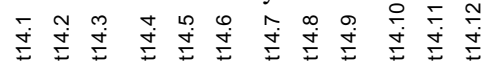

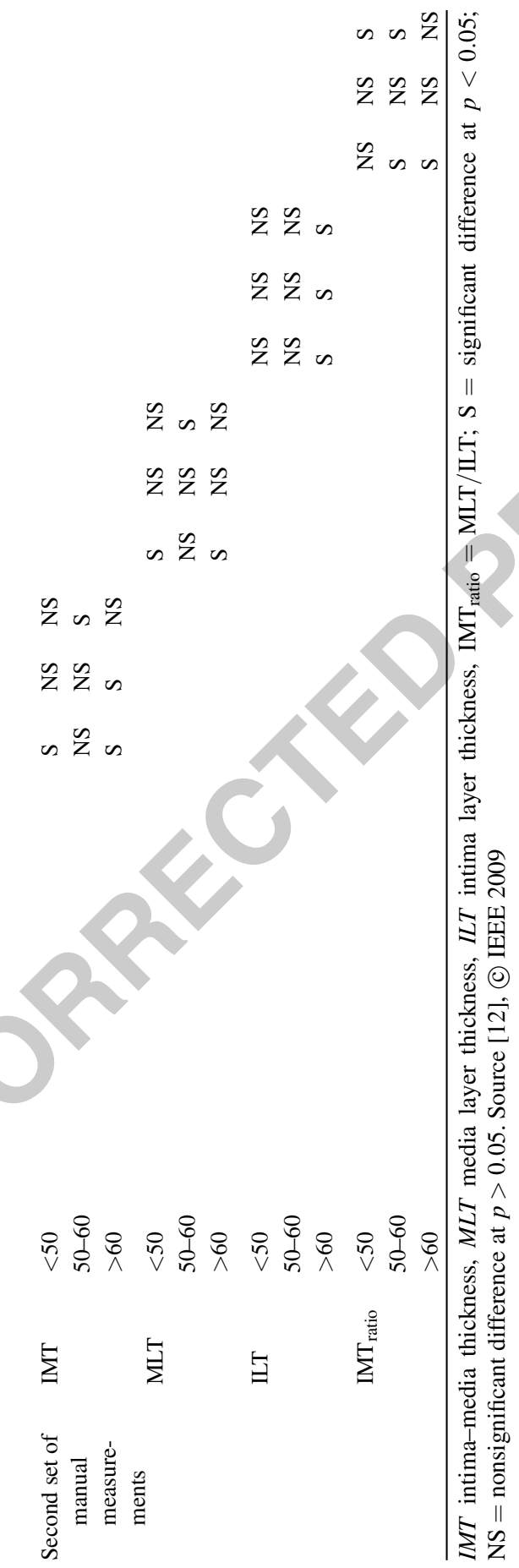




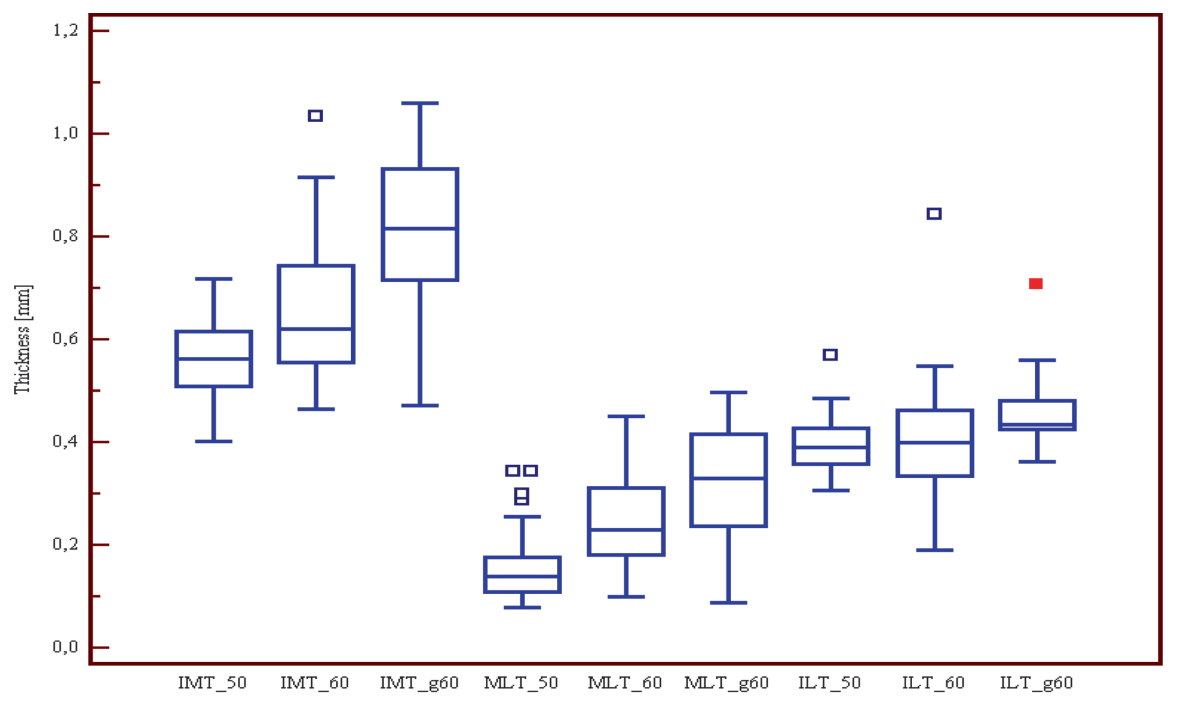

IQR: $0.1257,0.254,0.218 / 0.1577,0.872,0.1792 / 0.0949,0.1013,0.0573$

Fig. 2 Box plots for the manual measurements of the IMT, MLT, and ILT for the age groups below 50 (50), between 50 and 60 (60), and above 60 years (g60) old (i.e., IMT_50, ILT_60, and MLT_g60 represent the values below the age of 50 for IMC, ML, and IL, respectively). Source [12], (C) IEEE 2009

$F$-ratio of 11.6 with a corresponding significance level of $p=0.001$. This supports 357 the assumption of a linear relationship between age and MLT. The correlation 358 coefficient was $\rho=0.33$. It was shown that the MLT at the age of 55 is $0.25 \mathrm{~mm} 359$ and that the $95 \%$ confidence interval limits for the MLT are both $\pm 0.24 \mathrm{~mm}$. 360

The Bland-Altman plot between the first set manual measurements and the 361 snakes segmentation MLT measurements showed that the difference between the 362 manual and the snakes segmentation measurements was $-0.01 \mathrm{~mm}$ with limits of 363 agreement lying between $0.16 \mathrm{~mm}$ and $-0.18 \mathrm{~mm}$ (note that the pixel resolution was 364 $0.08 \mathrm{~mm}$ ). The Bland-Altman plot between the second set manual measurements 365 and the snakes segmentation MLT measurements showed that the difference 366 between the manual and the snakes segmentation measurements was $0.01 \mathrm{~mm}$ with 367 limits of agreement lying between $0.24 \mathrm{~mm}$ and $-0.23 \mathrm{~mm}$. The Bland-Altman 368 plot between the first and second set of manual measurements showed that the 369 difference between the first and the second set of the manual measurements was 370 $-0.02 \mathrm{~mm}$ with limits of agreement lying between $0.16 \mathrm{~mm}$ and $-0.19 \mathrm{~mm}$. There 371 was also a negative bias, as estimated by the mean difference, which showed that 372 on an average the snakes segmentation algorithm underestimates the area relative to 373 normal delineation.

Figure 2 presents box plots of the IMT, MLT, and ILT, for the three different 375 age groups, extracted from the manual IMC, ML, and IL segmentations for all 100376 images of the CCA for the three different age groups (below 50, between 50 and 60, 377 and above 60 years). 


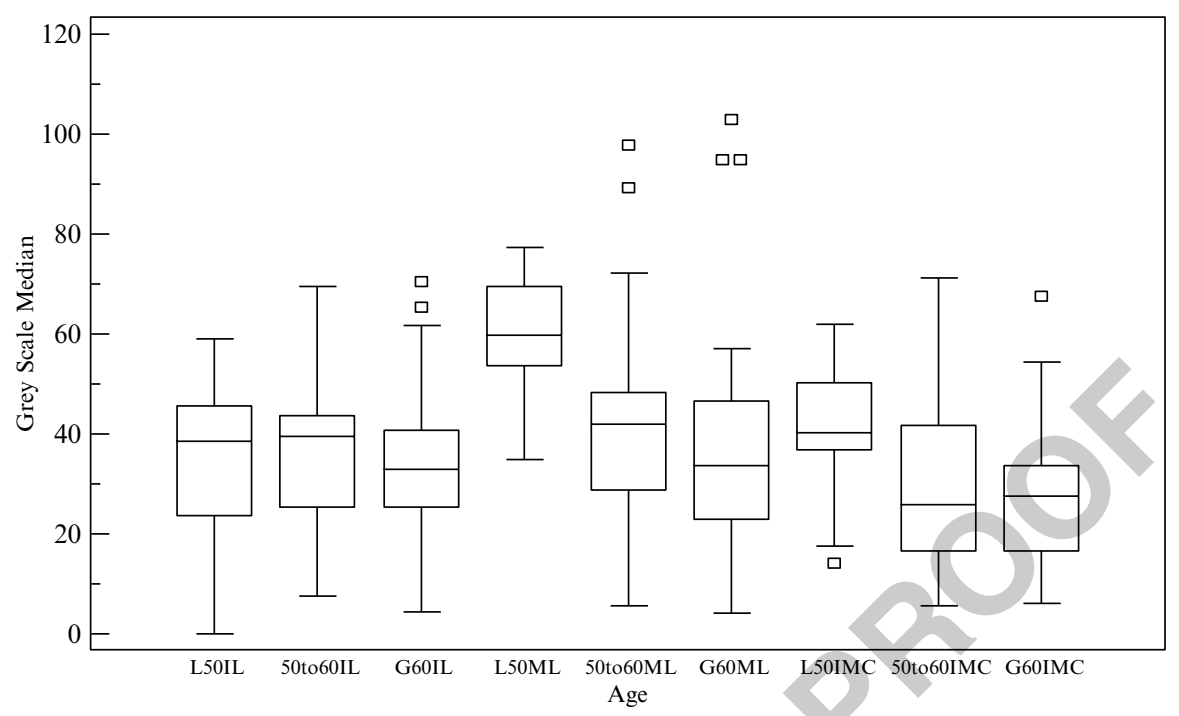

Fig. 3 Box plots of the gray-scale median (GSM) texture feature extracted from IL, ML, and IMC. Here we are using automated segmentations. In our description we let IQR denote the inter-quartile distance. From left to right, for the first three plots we have: L50IL for the IL for patients younger than $50(\mathrm{IQR}=22), 50$ to $60 \mathrm{IL}$ for the IL for patients from 50 to 60 years old $(\mathrm{IQR}=18.4)$, and G60IL for the IL for patients who are older than 60 years old (IQR $=15.4$ ). For the next three plots (middle), we have the same age groups for the ML and IQR values of 16, 19.5, and 23.7, respectively. The last three plots (leftmost) show the GSM values for the IMC with IQR values 13.6, 25.2, and 16.9, respectively. In each plot we display the median, lower, and upper quartiles and confidence interval around the median of the GSM. Straight lines connect the nearest observations with 1.5 of the inter-quartile range (IQR) of the lower and upper quartiles. Unfilled rectangles indicate possible outliers with values beyond the ends of the $1.5 \times \mathrm{IQR}$. Source [12], (C) IEEE 2009

Figure 3 presents box plots of the GSM texture features for the three different 379 age groups. These measurements were extracted using the automated IL/ML/IMC 380 segmentations.

Table 4 presents the median and inter-quartile range (IQR) of selected texture 382 features extracted from the automated IL, ML, and IMC segmentations of the 383 100 ultrasound images investigated. From Table 4, we can see that most of the 384 texture features extracted from the ML exhibit lower median and IQR values when 385 compared with those extracted from the IL and IMC (e.g.: mean, GSM, STD, 386 contrast, ASM, and coarseness).

In Table 5, we use the Wilcoxon rank sum test to compare selected texture 388 features extracted from automated and manual segmentations. We can see similar 389 results for both manual and automated segmentations. However, we note that there 390 are differences. To avoid issues associated with segmentation differences, we are 391 only interested in textural features that give significant differences for both manual 392 
Table 4 Texture features (median (IQR)) for the IL, ML, and IMC using automated segmentations

\begin{tabular}{llllll}
\hline & IL & ML & IMC & \\
\cline { 1 - 3 } Mean & $35(15.3)$ & $21(25)$ & $33(22)$ & \\
GSM & $35(18.5)$ & $28(18)$ & $30(21.3)$ & \\
STD & $16(6.6)$ & $14(7)$ & $16(5.7)$ & \\
Contrast & $52(75)$ & $28(32)$ & $61(57)$ & \\
DV & $16(27.8)$ & $81(56)$ & $32(32)$ & $\mathrm{t} 15.5$ \\
Complexity & $1,704(3,175)$ & $6,041(6,762)$ & $4,166(5,888)$ & $\mathrm{t} 15.7$ \\
ASM & $0.09(0.04)$ & $0.002(0.003)$ & $0.003(0.002)$ & $\mathrm{t} 15.8$ \\
Coarseness & $20(14.5)$ & $13(11)$ & $24(11.3)$ & $\mathrm{t} 15.9$ \\
SS-TEL & $38(33)$ & $78(53)$ & $56(38)$ & $\mathrm{t} 15.10$ \\
Entropy & $5.7(1.15)$ & $6(1.2)$ & $6.6(0.7)$ & $\mathrm{t} 15.11$ \\
Roughness & $2.46(0.187)$ & $2.2(0.100)$ & $2.238(0.079)$ & $\mathrm{t} 15.12$ \\
Periodicity & $0.8(0.07)$ & $0.9(0.06)$ & $0.8(0.2)$ & $\mathrm{t} 15.13$
\end{tabular}

IQR inter-quartile range, GSM gray-scale median, $S T D$ standard deviation, $D V$ difference variance, $A S M$ angular second moment, SS-TEL SS-texture energy laws. Source [11], (C) CMIG 2009

Table 5 Wilcoxon rank sum comparison tests performed on texture features (first column) extracted from the IL, ML, and IMC using manual (M) and automated (A) segmentations. In boldface, we have the texture features identified by the automated segmentation method that also exhibits significant differences for manual segmentation

\begin{tabular}{|c|c|c|c|c|c|c|c|}
\hline & \multicolumn{3}{|c|}{ Manual (M) } & \multicolumn{3}{|c|}{ Automated (A) } & \multirow{2}{*}{$\begin{array}{l}\sharp 16.1 \\
\mathrm{t} 16.3\end{array}$} \\
\hline & IL-ML & IL-IMC & ML-IMC & IL-ML & IL-IMC & ML-IMC & \\
\hline Mean & $\mathrm{S}(0.01)$ & $\mathrm{NS}(0.5)$ & $S(0.001)$ & S (0.02) & NS $(0.81)$ & $S(0.004)$ & 16.4 \\
\hline GSM & NS (0.39) & NS (0.62) & $S(0.001)$ & NS $(0.3)$ & NS $(0.45)$ & $\mathrm{S}(\mathbf{0 . 0 4 )}$ & 16.5 \\
\hline Stand. Dev. & $\mathrm{S}(0.01)$ & $\mathrm{S}(0.001)$ & $S(0.001)$ & S (0.001) & NS $(0.1)$ & $\mathrm{S}(\mathbf{0 . 0 0 1 )}$ & $\mathrm{t} 16.6$ \\
\hline Contrast & $\mathrm{S}(0.001)$ & $S(0.001)$ & $S(0.001)$ & $S(0.001)$ & $\mathrm{NS}(0.23)$ & $S(0.001)$ & $\mathrm{t} 16.7$ \\
\hline Diff. Var. & $\mathrm{S}(0.001)$ & $\mathrm{S}(0.001)$ & $\mathrm{S}(0.01)$ & $S(0.001)$ & S (0.007) & NS (0.09) & t16. \\
\hline Complexity & $S(0.001)$ & $\mathrm{S}(0.001)$ & $S(0.04)$ & S (0.001) & $S(0.001)$ & NS (0.09) & $\mathrm{t} 16.9$ \\
\hline ASM & $\mathrm{NS}(0.3)$ & $\mathrm{S}(0.001)$ & NS (0.13) & S (0.004) & $S(0.001)$ & $S(0.001)$ & $\mathrm{t} 16$ \\
\hline Coarseness & $S(0.001)$ & $S(0.021)$ & $\mathrm{S}(0.001)$ & $S(0.001)$ & $S(0.005)$ & $S(0.001)$ & $\mathrm{t} 16$ \\
\hline SS-TEL & $S(0.009)$ & $S(0.009)$ & NS (0.13) & $\mathrm{S}(\mathbf{0 . 0 0 1 )}$ & $S(0.02)$ & NS (0.18) & $\mathrm{t} 16.1$ \\
\hline Entropy & $\mathrm{S}(0.017)$ & $\mathrm{S}(0.001)$ & NS $(0.85)$ & S (0.001) & $S(0.001)$ & $\mathrm{S}(\mathbf{0 . 0 0 8 )}$ & $\mathrm{t} 16$. \\
\hline Periodicity & $\mathrm{S}(0.001)$ & NS (0.92) & $\mathrm{S}(0.001)$ & $\mathrm{S}(\mathbf{0 . 0 0 1 )}$ & NS (0.07) & $S(\mathbf{0 . 0 2})$ & 16. \\
\hline
\end{tabular}

$I L$ intima layer, $M L$ media layer, $I M C$ intima-media complex. The $p$ value is shown in parentheses ( $\mathrm{S}=$ significantly different at $p<0.05, \mathrm{NS}=$ nonsignificantly different at $p \geq 0.05)$. Source [11],

(C) CMIG 2009

and automated segmentations. For better visualization, we put these features in bold 393 face (see Table 5). Between the IL and the ML, we have significant differences in 394 10 out of 11 features. Between the IL and the IMC, we have six texture features 395 with significant differences. Similarly, we have eight texture features that exhibit 396 significant differences between the IL and the ML in Table 5. We refer to Table 6 for 397 a summary of the texture feature values. 
Table 6 Texture characteristics of IL vs. ML based on the texture feature values given in Table 1

\begin{tabular}{|c|c|c|}
\hline $\begin{array}{l}\text { Corresponding } \\
\text { features from Table } 1\end{array}$ & IL & ML \\
\hline Mean, GSM & Brighter & Darker \\
\hline Contrast, ASM & Higher contrast & Less contrast \\
\hline Complexity, entropy & Low complexity & High complexity \\
\hline Coarseness & $\begin{array}{l}\text { More coarse, i.e., large } \\
\text { areas with small } \\
\text { gray tone variations }\end{array}$ & $\begin{array}{l}\text { Less coarse, i.e., less } \\
\text { local uniformity in } \\
\text { intensity }\end{array}$ \\
\hline Roughness & Slightly rougher & Smoother \\
\hline Periodicity & $\begin{array}{l}\text { Less periodical, more } \\
\text { heterogeneous }\end{array}$ & $\begin{array}{l}\text { More periodical, more } \\
\text { homogeneous }\end{array}$ \\
\hline
\end{tabular}

Source [11], (c) CMIG 2009

Table 7 presents the Mann-Whitney rank sum test performed on selected texture 399 features extracted from the automated IL, ML, and IMC segmentations for the three 400 different age groups. It is shown that some of the texture features (GSM, STD, DV, 401 complexity, coarseness, and SS-TEL) exhibit significantly different values between 402 different age groups.

\section{Discussion}

In this study, both manual and automated IMT, MLT, and ILT measurements are 405 reported for 100 longitudinal ultrasound images of the CCA and their variation with 406 age and sex. The importance of the CCA ML for the evaluation of the risk of CVD 407 was outlined in [2] and shown in [15], that the MLT was thinner for this group of 408 subjects. Also it was shown in [16] that the MLT was strongly related with age for 409 the radial and anterior tibial arteries. It was also found (see Table 6) that the ML 410 is less dark, has less contrast, is more periodic, and is less coarse compared to IL. 411 We have found a decrease of ML GSM with increasing age, as well as decrease 412 with thickness (MLT), that can be attributed to the fact that, at the initial stages of 413 atherosclerosis disease, there is an increased concentration of lipids and hyperplasia 414 of muscle fibers in the ML, which produces hypoechoic (echolucent) structures. It 415 was also shown that the GSM of male patients is brighter than that of the female 416 patients.

Table 1 showed that the manual IMT measurements are larger and have a larger 418 range of values, whereas the first set of manual and the automated MLT measure- 419 ments are almost the same, while the second set of manual measurements were 420 higher. Similar IMT findings were also reported in [9, 10, 14]. The manual IMT mean 421 $\pm \mathrm{SD}$ measurements reported in this study $(0.71 \pm 0.17 \mathrm{~mm}$ and $0.72 \pm 0.17 \mathrm{~mm}$ for 422 manual first and second sets, respectively) were larger than the snakes segmented 423 measurements $(0.67 \pm 0.12 \mathrm{~mm})$, and this finding was also reported in other studies. 424 More specifically, the manual vs. the snakes segmented IMT measurements reported 425 


\section{Editor's Proof}

116

C.P. Loizou et al.

-N⿴囗十丁

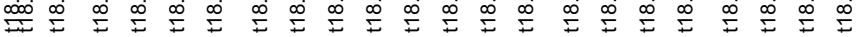

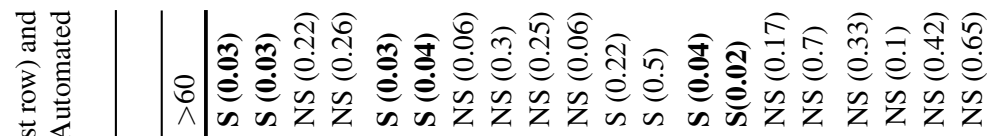

논

ํㅝㅀ

을

है

$\stackrel{0}{\leftrightarrows}$

$3:=$

这

$\stackrel{0}{\approx}$

कू

क्ष

플

:

tै

巳

约

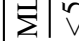

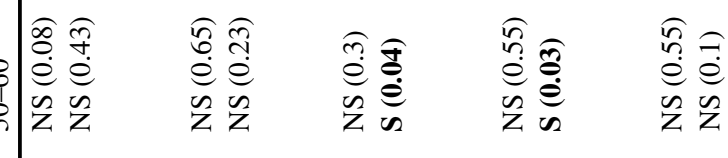

ㄴํำ

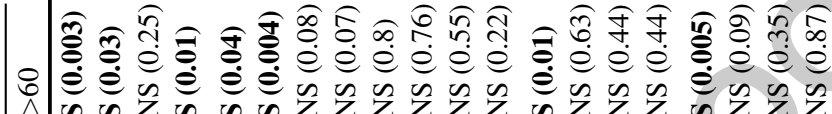

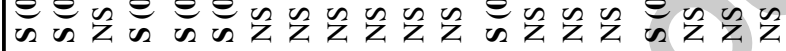

స

$\sum \sum^{\pi}$

$\hat{\theta} \pm$

$\stackrel{乛}{\Xi}$

疎

焉

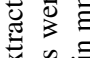

×

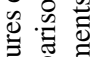

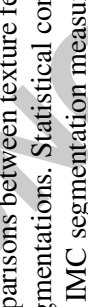

言

สำ

节完

क 政

츨

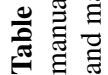

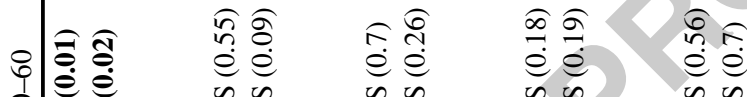

in is

乙望

乙 $气$

乙

乙望

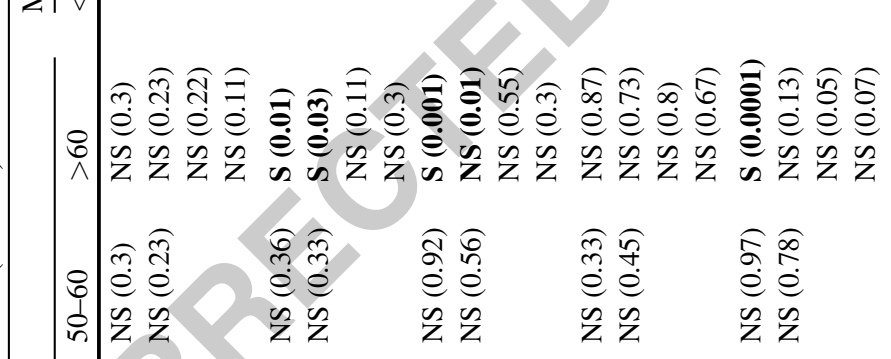

$=1 \vee$

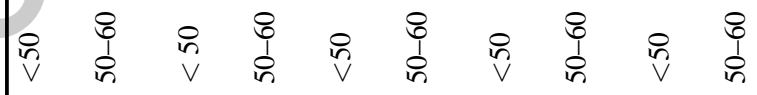

$\sum_{\substack{0 \\ 0}}$

宣

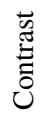

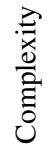

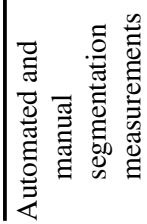




\section{Editor's Proof}

Media and Intima Thickness and Texture Analysis of the Common Carotid Artery

Б)

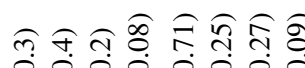

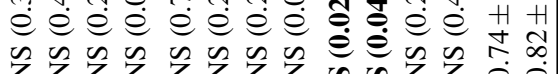

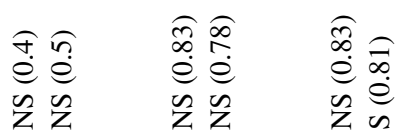

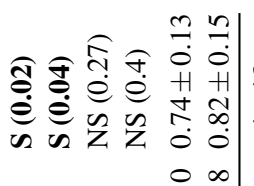

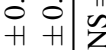

$\infty \begin{aligned} & 0 \\ & 0\end{aligned}$

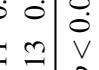

H

in

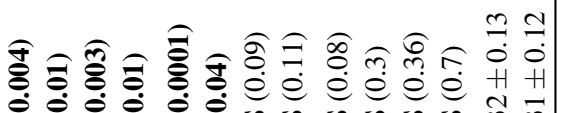

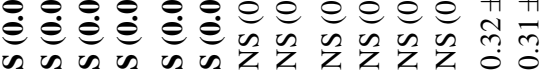

เก

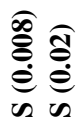

ले

e

乙

eे

乙约

$\circ \infty$

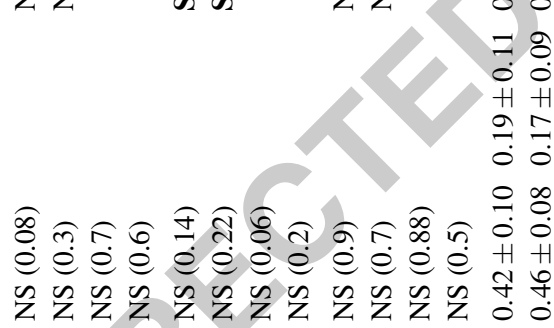

0 o

กิ

$=$ oे

요

$\div \circ$

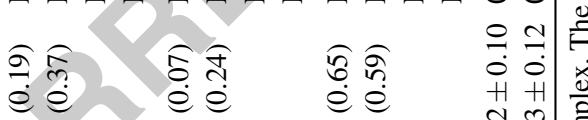

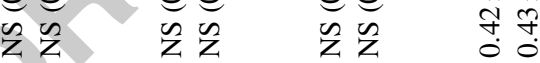

으. ㄴ. 궁

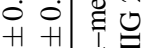

ำ ำ

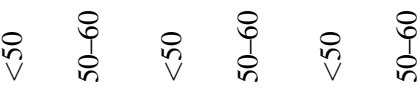

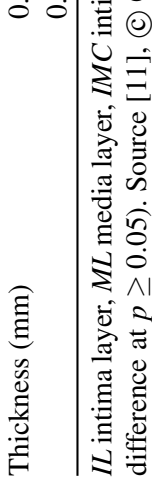


in other studies were $(0.93 \mathrm{~mm} v s .0 .88 \mathrm{~mm})$ [9], $(0.72 \mathrm{~mm}$ vs. $0.63 \mathrm{~mm})$ [13], 426 $(0.8 \mathrm{~mm}$ vs. $0.7 \mathrm{~mm})$ [14], and $(0.92 \mathrm{~mm}$ vs. $0.88 \mathrm{~mm})$ [29]. There is a large variation 427 in the IMT measurements between the different studies because different material 428 and different measuring methodologies were used.

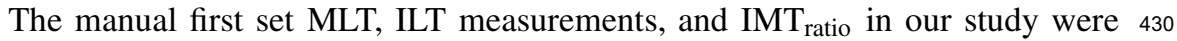
$0.25 \pm 0.12 \mathrm{~mm}, 0.43 \pm 0.10 \mathrm{~mm}$, and $0.5834 \pm 0.323 \mathrm{~mm}$, respectively (similar 431 values were obtained for the second set of manual measurements and the automated 432 measurements). In [15], the manual IMT, MLT, and ILT measurements carried 433 out for the CCA by an expert using high-resolution ultrasound with a broadband 434 probe and a $25 \mathrm{MHz}$ center frequency, between subjects with and without CVD, 435 were $0.75, \pm 0.17 \mathrm{~mm}$ vs. $0.72 \pm 0.20 \mathrm{~mm}(p=0.4), 0.45 \pm 0.14 \mathrm{~mm}$ vs. $0.38 \pm 436$ $0.16 \mathrm{~mm}(p<0.05)$, and $0.17 \pm 0.006 \mathrm{~mm}$ vs. $0.22 \pm 0.06 \mathrm{~mm}(p<0.0001), 437$ respectively. It was shown in [15] that subjects with CVD (coronary heart disease, 438 myocardial infarction, or stroke) had a significantly thicker IL and a thinner ML than 439 healthy subjects. The IMT $\mathrm{I}_{\text {ratio }}$ also differed significantly between subjects with and 440 without a diagnosis of CVD $(0.43 \pm 0.20$ vs. $0.75 \pm 0.48, p=0.0002)$. The MLT 441 and ILT were manually measured from the radial and tibial arteries in 90 subjects $\left(\begin{array}{ll}10 & 442\end{array}\right.$ to 90 years old), using a $55 \mathrm{MHz}$ two-dimensional ultrasound transducer [16]. The 443 values reported for the MLT ranged from $0.159 \pm 0.039 \mathrm{~mm}$ to $0.227 \pm 0.038 \mathrm{~mm}, 444$ whereas for the ILT the values ranged from $0.49 \pm 0.08 \mathrm{~mm}$ to $0.81 \pm 0.019 \mathrm{~mm}, 445$ respectively. Finally, in [8], it was found that the median MLT of muscular arteries 446 was $0.6 \mathrm{~mm}$ in the absence of a lesion, decreasing to $0.1 \mathrm{~mm}$ in the area of maximal 447 atherosclerosis. The in vivo study was performed in 29 patients undergoing coronary 448 or peripheral vascular procedures using intravascular ultrasound with a $30 \mathrm{MHz} 449$ frequency ultrasound probe.

It is shown from Table 1 that for the MLT measurements, the standard deviation, 451 sd, for both the manual ( 0.12 and 0.14 for the first and second set of manual mea- 452 surements, respectively) and the automated $(0.11)$ and the coefficient of variation, 453 CV\% (34\% and 9.8\% for the first and second set of manual measurements, and 454 $12.3 \%$ for the automated measurements), are large. This is explained due to the fact 455 that the ML is very small with complex texture and, therefore, very difficult to be 456 estimated correctly either manually or automatically.

Table 1 also shows that the inter-observer errors, se, for the IMT and the MLT, 458 are larger for the manual segmentation measurements (for both the first set and 459 the second set of manual measurements). Also, the results from Table 1 showed 460 that high intra-observer variabilities occur when manual measurements are made. 461 Smaller values for se were obtained for the automated segmentation measurements, 462 indicating a better performance of the automated vs. the manual measurements as 463 documented in $[10,22]$.

Table 2 showed that no significant differences were found between men and 465 women for the IMT, MLT, ILT, and the $\mathrm{IMT}_{\text {ratio }}$, as also shown in [15] and that 466 the $\mathrm{IMT}_{\text {ratio }}$ did not differ significantly between male and female groups. $\quad 467$

The nonsignificant (NS) differences between the manual and the automated 468 segmentation method, estimated by the Wilcoxon rank sum test show that the 469 manual measurements may be replaced by the snakes segmentation measurements 470 
with confidence. It was also shown that the manual measurements of the expert at 471 time 0 vs. time 6 months were NS. The correlation coefficient, $\rho$, showed that the 472 strongest relationship existed between the MLT manual first set and the automated 473 segmentation measurements (with $\rho=0.72$ ), where higher values were reported. 474 The Wilcoxon rank sum test performed in Table 3 showed that the first set of 475 manual and automated IMT measurements between all the age groups are mostly 476 significantly different (S), whereas the manual and automated measurements of the 477 MLT and ILT are mostly nonsignificantly different (NS). Furthermore, the Wilcoxon 478 rank sum test for the $\mathrm{IMT}_{\text {ratio }}$ showed that there are no clear statistical significance 479 between the different age groups; therefore, this measure may not be used as an 480 indicative value for atherosclerotic disease with increasing age.

Based on the Bland-Altman test a small discrepancy, of around $0.01 \mathrm{~mm}$, was 482 found between the manual and the automated MLT measurements. On this basis the 483 two methods (manual and snakes segmentation) can be considered interchangeable. 484 There are no other studies reported in the literature, where Bland-Altman plots were 485 used for comparing the manual and automated MLT measurements. The Bland- 486 Altman plot was used in [29], where 24 carotid ultrasound images were analyzed by 487 two experts for validating the results of IMT measurements using a newly developed 488 system by comparing them with those obtained using previous methods and showed 489 no evidence of bias between the two methods.

This study shows that there might be a linear relationship between MLT 491 automated measurements and age, where all measurements showed an increase with 492 age. Similar findings were also reported for the IMT in [10]. It was also shown 493 that in this study the values of the mean IMT in a carotid artery vary between 494 $0.54 \mathrm{~mm}$ and $0.88 \mathrm{~mm}$, depending on age, and this is also consistent with other 495 studies $[2,6,14,28]$, whereas the values of the MLT vary between $0.13 \mathrm{~mm}$ and 496 $0.37 \mathrm{~mm}$. Furthermore, in [16], the IMT, MLT, and ILT of 90 healthy subjects (aged 497 between 10 and 90 years), were manually measured at their radial and anterior tibial 498 artery. It was shown that age was strongly related with IMT, MLT, and ILT of both 499 peripheral arteries.

Similar to [37], we have also found that the IMT is generally larger in men than 501 in women. Furthermore, in agreement with $[28,36]$, we have also found that the 502 IMT increases linearly with age.

In [43], increased IMT has been found in young adults with childhood-onset 504 chronic kidney disease (CKD). The disease stage at which these patients first 505 develop abnormalities of arterial texture is unknown. It was also shown that in 55506 children with stages 2 to $4 \mathrm{CKD}$, thickening of IMT occurs early in the course 507 of the disease and is most prominently marked in dialyzed patients. Furthermore, 508 morphologic alterations were found in both muscular and elastic type arteries as 509 early as in the second decade of life. The degree of thickening depends on the degree 510 of renal dysfunction. The authors also found that $61 \%$ of children with stages 2 to 4511 CKD had IMT equal to or exceeding the 95th percentile for age. 512

It was shown in [33] that the GSM of the IM layer is the earliest change 513 representing atherosclerotic disease in the arterial wall that can currently be imaged 514 in vivo. This may be the first marker of atherosclerosis and may precede the 515 
development of significant increase in IMT. This would enable earlier identification 516 of high-risk individuals based on the analysis of the CCA artery wall textural 517 characteristics.

In [34], the texture features of 12 subjects ( $28 \pm 2$ years) with familial hy- 519 percholesterolemia $(\mathrm{FCH})$ and without $\mathrm{CVD}$, before and 3 months after treatment 520 with atorvastatin were investigated, where the entropy and the ASM were extracted 521 from the IMC band. Before treatment of atorvastatin significantly higher entropy 522 (normal: $0.082 \pm 0.02$ vs. FCH : $0.57 \pm 0.12$ ) and lower ASM (normal: $0.01 \pm 523$ 0.0011 vs. FCH : $0.004 \pm 0.0001)$ values were found when compared to the normal 524 tissue.

The normalization method used in this study was documented to be helpful in 526 the manual contour extraction [25] as well as the snakes segmentation of the IMC 527 [10] and the atherosclerotic carotid plaque segmentation [26] and texture analysis 528 [11]. Moreover, this method increased the classification accuracy of different plaque 529 types as assessed by the experts [27]. Ultrasound image normalization was carried 530 out, prior to segmentation of the IMT in [24], where histogram equalization was 531 performed on carotid artery ultrasound images for increasing the image contrast.

\subsection{Study Limitations}

It has been demonstrated in $[2,31]$ that the blood-endothelium (intima) interface 534 and the media-adventitia interface correspond to their anatomical/histological 535 counterparts. This is not the case with the intima-media interface because the width 536 of the white band closer to the lumen is gain dependent and, therefore, variable. 537 Thus the measurement of the MLT represents only an ultrasonic measurement (as 538 also discussed above). It should be noted, however, that part of the ML, shown 539 in ultrasound image as an echolucent band (Fig. 1a, band Z6), contains the hypo 540 echoic muscular arterial tunica media and perhaps hypo echoic part of the arterial 541 tunica intima. It is well known that atherosclerosis causes an increase of the arterial 542 tunica intima thickness. As has been also reported earlier in the literature [13], 543 the histological boundaries between the arterial tunica intima and media (internal 544 elastic lamina) in ultrasound images cannot be accurately delineated. Therefore, we 545 hypothesize that the increase of the MLT with age as reported in our study (see also 546 [10]) may be a misleading finding, since part of the ML, as shown in ultrasound 547 images, may belong to the arterial tunica intima. We recommend that the above 548 hypothesis should be taken into account in future studies aiming in studying the 549 ML texture and morphology. It should be furthermore noted that there are no other 550 studies reported in the literature where ML segmentation and measurements were 551 performed on longitudinal ultrasound images of the CCA.

While IMT is an established measure of the vascular wall disease [2], GSM is 553 less commonly used while other features have not been used before. GSM analysis 554 has previously mainly been performed on plaques and then found to be related to 555 histological features of the plaque, such as the elastin and calcium content, as well 556 as to the size of the lipid-rich necrotic core [44]. However, the histological correlate 557 
to variation in the ML texture features has to be evaluated and the prognosis impact 558 of these new variables has to be investigated. It has also been observed that there 559 is an increase in the granularity in association with atherosclerotic disease [31]. 560 A granular IMC indicates more advanced atherosclerosis, which may precede the 561 development of significant IMT thickening.

A limitation of the proposed method is that the manual and automated ML and IL 563 segmentation measurements were performed in linear segments of the CCA because 564 in the clinical praxis, the experts are delineating the IMC and the ML only in those 565 parts of the vessel where there are no significant artifacts, signal drop outs, and 566 structure irregularities $[2,9,10,20,27,35]$.

Another limitation of the proposed method is the presence of acoustic shadowing 568 together with strong speckle noise, which hinders the visual and automatic analysis 569 in ultrasound images [9, 10]. Such images as well as images with extensive 570 echolucency and calcification, where the ML was not well visually recognized, were 571 excluded from the study. Backscattered ultrasound is also angle dependent. During 572 the recording of the images, a standard recording technique was used to adjust the 573 position of the probe so that the ultrasound beam was at right angles to the arterial 574 wall. This improved the IMC visualization. Moreover, the new spatial compound 575 imaging technique might optimize further carotid ultrasound imaging [25, 27]. Due 576 to the small size of the ML and the estimation and positioning of the initial snake 577 contour may sometimes result to segmentation errors. This should be placed as close 578 as possible to the area of interest, otherwise it may be trapped into local minima or 579 false edges and converge to a wrong location.

As it has already been mentioned in Sect. 2.1, the axial resolution of the system 581 used was $0.11 \mathrm{~mm}$, which indicates that structures greater than $0.11 \mathrm{~mm}$ can be 582 correctly visualized and measured (see also [32]). In addition, it is noted that the 583 pixel resolution was $0.06 \mathrm{~mm}$. For structures thinner than $0.11 \mathrm{~mm}$, the two echo 584 interfaces (i.e., of ML) cannot be separated and measurements of these structures are 585 not possible. For the dataset investigated in this study, $6 \%$ of the measurements were 586 below $0.11 \mathrm{~mm}$; therefore, these measurements may be considered as unreliable. It 587 should be noted, however, that with new ultrasound equipment and new probes, that 588 are available having a recording frequency in the range of $15 \mathrm{MHz}$, this problem will 589 be highly limited. In another study where the variability of the IMT between experts 590 and between the manual and the automated IMT measurements was investigated 591 [32], it was shown that the reproducibility of the IMT measurements may be further 592 improved if the IMT mean value is computed from multiple ultrasound images 593 (frames) of the carotid, instead of the mean value obtained from only one frame 594 [32]. These can be also investigated in a future study for the case of the MLT and 595 the ILT measurements.

In the present study, in less than $8 \%$ of the cases, the positioning of the initial 597 snake contour was not calculated correctly. Furthermore, there were also another $6 \% 598$ of the cases where the ML structure was very small and the two snake contours were 599 trapped together. For these cases the user of the proposed system may run the snakes 600 initialization procedure again in order to estimate the correct initial snake contour, 601 and during the snakes deformation the user may interact and manually correct the 602 contour. 


\section{Concluding Remarks and Future Work}

It was clearly illustrated in this study that both manual and automated IMT, MLT, 605 and ILT measurements could be carried out successfully. Also, it was shown that 606 there was overall no significant difference between the manual and the automated 607 measurements, between the male and the female subjects, as well as that these 608 increase with age.

The segmented IMC, ML, and IL bands may be used for extracting texture 610 features [30], which might be able to differentiate between subjects with high and 611 low risk of stroke. Furthermore, the change of these textural characteristics with 612 age may give additional information for characterizing subjects in different risk 613 groups. In a recent study [35], it was shown that the gray-scale median of the 614 IMC of the CCA is closely related to the echogenecity in overt carotid plaques. 615 This finding suggests that the gray-scale median and other texture features extracted 616 from the IMC or the ML bands could have prognostic impact for the assessment 617 of cardiovascular risk. However, the $\mathrm{IMT}_{\text {ratio }}$ was not found to be statistically of 618 significant difference with increasing age.

Our texture analysis showed that: (a) there are significant differences between 620 some texture features extracted from the IL, ML, and IMC (mean, gray-scale median 621 (GSM), standard deviation, contrast, difference variance, and periodicity), (b) some 622 of the texture features can be associated with the increase (difference variance, 623 entropy) or decrease (GSM) of patient's age, (c) the GSM of the ML falls linearly 624 with increasing ML thickness (MLT) and with increasing age, (d) the GSM of male 625 subjects is larger than that of female subjects (se, Fig. 5.4), and (e) male and female 626 subjects may be better distinguished using texture features extracted from the IMC. 627

Risk factors like smoking, blood pressure, inflammation markers, and cholesterol 628 correlate to the traditional carotid IMT $[1,3,4,16]$. It would have been valuable to 629 relate these risk factors to AM-FM analysis and not only the age as done in this 630 study. Ongoing studies from our group will give more data on this topic. 631

Future work will investigate whether it is possible to identify a group of patients 632 at risk of atherosclerosis based on their texture features extracted from the IL, the 633 ML, and the IMC of high-resolution ultrasound images of the CCA. It may also 634 be possible to identify and differentiate those individuals into high- and low-risk 635 groups according to their cardiovascular risk before the development of plaques. 636 The proposed methodology may also be applied to a group of people, which already 637 developed plaques in order to study the contribution of the ML texture features 638 to cardiovascular risk. Both groups of patients may benefit by prognosing and 639 managing future cardiovascular events. Another possible future application of the 640 proposed methodology is that it can be used to investigate possible effects of statins 641 or other drugs in texture feature changes of the ML of the CCA.

The methodology presented in this study will be further evaluated on ultrasound 643 images of the CCA collected on a large-scale epidemiological study by our group as 644 well as for the segmentation and measurement of curved segments of the bifurcation 645 bulb of the CCA. 
Credit/copyright notice: Based on "Manual and automated media and intima 647 thickness measurements of the common carotid artery," by Loizou CP, Pattichis CS, 648 Nicolaides A, Pantziaris M (2009) IEEE Trans Ultrason Ferroelectr Freq Control 649 56(5):983-994, (C) 2009EEE, and on "Ultrasound image texture analysis of the 650 intima and media layers of the common carotid artery and its correlation with age 651 and gender" by Loizou CP, Pantziaris M, Pattichis MS, Kyriakou E, Pattichis CS 652 (2009) Comput Med Imaging Graph 33(4):317-324, (c) CMIG2009.

\section{References}

1. American Heart Association (2007) Heart disease and stroke statistics-2008, update, Dallas. 655 http://www.americanheart.org/presenter.jhtml.

2. Pignoli P, Tremoli E, Poli A, Oreste P, Paoleti R (1986) Intima plus media thickness of the 657 arterial wall: a direct measurement with ultrasound imaging. Atherosclerosis 74(6):1399-1406 658

3. Touboul P-J, Labreuche J, Vicaut E, Amarenco P (2005) Carotid intima-media thickness, 659 plaques, and Framingham risk score as independent determinants of stroke risk. Stroke 660 36(8):1741-1745

4. Watanabe T, Koba S, Kawamura M, Itokawa M, Idei T, Nakagawa Y, Iguchi T, Katagiri T 662 (2004) Small dense low-density lipoprotein and carotid atherosclerosis in relation to vascular 663 dementia. Metabolism 53(4):476-482

5. Mario CD, Gorge G, Peters R, Pinto F, Hausmann D, von Birgelen C, Colombo A, Murda H, 665 Roelandt J, Erbel R (1998) Clinical application and image interpretation in coronary ultra- 666 sound. Study group of intra-coronary imaging of the working group of coronary circulation 667 and of the subgroup of intravascular ultrasound of the working group of echocardiography of 668 the European Society of Cardiology. Eur Heart J 19(2):201-229 669

6. Grønhold ML, Nordestgaard BG, Schroeder TV, Vorstrup S, Sillensen H (2001) Ultrasonic 670 echolucent carotid plaques predict future strokes Circulation 104(1):68-73 671

7. Wilhjelm JE, Grønholdt MLM, Wiebe B, Jespersen SK, Hansen LK, Sillensen H (1998) 672 Quantitative analysis of ultrasound B-mode images of carotid atherosclerotic plaque: cor- 673 relation with visual classification and histological examination. IEEE Trans Med Imaging 674 17(6):910-922

8. Gussenhoven EJ, Frietman PA, van Suylen SHRJ, van Egmond FC, Lancee CT, van Urk H, 676 Roelandt JR, Stijnen T, Bom N (1991) Assessment of medial thinning in atherosclerosis by 677 intravascular ultrasound Am J Cardiol 68:1625-1632 678

9. Wendelhag I, Liang Q, Gustavsson T, Wikstrand J (1997) A new automated computerized 679 analysing system simplifies reading and reduces the variability in ultrasound measurement of 680 intima media thickness. Stroke 28:2195-2200

10. Loizou CP, Pattichis CS, Pantziaris MS, Tyllis T, Nicolaides AN (2007) Snakes based 682 segmentation of the common carotid artery intima media. Med Biol Eng Comput 45:35-49 683

11. Loizou CP, Pantziaris M, Pattichis MS, Kyriakou E, Pattichis CS (2009) Ultrasound image 684 texture analysis of the intima and media layers of the common carotid artery and its correlation 685 with age and gender. Comput Med Imaging Graph 33(4):317-324 686

12. Loizou CP, Pattichis CS, Nicolaides A, Pantziaris M (2009) Manual and automated media and 687 intima thickness measurements of the common carotid artery. IEEE Trans Ultrason Ferroelectr 688 Freq Control 56(5):983-994

13. Gutierrez M, Pilon P, Lage S, Kopel L, Carvalho R, Furuie S (2002) Automatic measurement 690 of carotid diameter and wall thickness in ultrasound images. Comput Cardiol 29:359-362 691

14. Delsanto S, Molinari F, Giustetto P, Liboni W, Badalamenti S, Suri JS (2007) Characterization 692 of a completely user independent algorithm for carotid artery segmentation in 2-D ultrasound 693 images. IEEE Trans Instrum Meas 56(4):1265-1274 
15. Rodriguez-Maciasa KA, Lindbc L, Naessena T (2006) Thicker carotid intima layer and thinner 695 media layer in subjects with cardiovascular diseases: an investigation using noninvasive high- 696 frequency ultrasound. Atherosclerosis 189(2):393-400

16. Osika W, Dangardt F, Grönros J, Lundstam U, Myredal A, Johansson M, Volkmann R, 698 Gustavsson T, Gan LM, Friberg P (2007) Increasing peripheral artery intima thickness from 699 childhood to seniority. Arterioscler Thromb Vasc Biol 27:671-676

17. Mintz GS, Nissen SE, Anderson W, Bailey SR, Erbel R et al (2001) American college of 701 cardiology clinical expert consensus document on standards for acquisition, measurements and 702 reporting intravascular ultrasound studies (IVUS). J Am Coll Cardiol 37:1478-1492 703

18. Loizou CP, Pattichis CS, Pantziaris M, Nicolaides A, Georgiou N, KyriakouE (2007) Media 704 thickness measurement of the common carotid artery. Proceedings of the 29th annual interna- 705 tional conference of the IEEE engineering in medicine and biology society, Cite Int., Lyon, 706 FrP1B6.5, 23-26 Aug 2007

19. Kyriakou E, Pattichis MS, Christodoulou CH, Pattichis CS, Kakkos S, Griffin M, Nicolaides 708 AN (2005) Ultrasound imaging in the analysis of carotid plaque morphology for the assessment 709 of stroke. In: Suri JS, Yuan C, Wilson DL, Laxminarayan S (eds) Plaque imaging: pixel to 710 molecular level, IOS, pp 241-275

20. Tegos TJ, Sabetai MM, Nicolaides AN, Elatrozy TS, Dhanjil S, Stevens JM (2001) Patterns 712 of brain computed tomography infraction and carotid plaque echogenicity. J Vasc Surg 713 33:334-339

21. Williams DJ, Shah M (1992) A fast algorithm for active contour and curvature estimation. 715 Comput Vis Image Underst 55(1):4-26

22. Chalana V, Kim Y(1997) A methodology for evaluation of boundary detection algorithms on 717 medical images. IEEE Trans Med Imaging 16(5):642-652

23. Bland JM, Altman DG (1986) Statistical methods for assessing agreement between two 719 methods of clinical measurement. Lancet 1(8476):307-310

24. Mojsilovic A, Popovic M, Amodaj N, Babic R, Ostojic M (1997) Automatic segmentation of 721 intravascular ultrasound images: a texture based approach. Ann Biomed Eng 25:1059-1071 722

25. Loizou CP, Pattichis CS, Pantziaris MS, Tyllis T, Nicolaides AN (2006) Quantitative quality 723 evaluation of ultrasound imaging in the carotid artery. Med Biol Eng Comput 44(5):414-426 724

26. Loizou CP, Pattichis CS, Pantziaris MS, Nicolaides AN (2007) An integrated system for the 725 segmentation of atherosclerotic carotid plaque. IEEE Trans Inf Technol Biomed 11(5):661-667 726

27. Nicolaides AN, Kakkos SK, Griffin M, Sabetai M, Dhanjil S, Thomas D et al (2005) Effect of 727 image normalization on carotid plaque classification and the risk of ipsilateral hemispheric 728 events: results from the asymptomatic carotid stenosis and risk of stroke study. Vascular 729 1(4):211-221

28. Graf S, Gariery J, Massonneau M, Armentano R, Mansour S, Barra J, Simon A, Levenson 731 J (1999) Experimental and clinical validation of arterial diameter waveform and intimal 732 media thickness obtained from B-mode ultrasound image processing Ultrasound Med Biol 733 25(9): 1353-1363

29. Mancini GBJ, Abbott D, Kamimura C, Yeoh E (2004) Validation of a new ultrasound method 735 for the measurement of carotid artery intima medial thickness and plaque dimensions. Can J 736 Cardiol 20(13)1355-1359

30. Christodoulou CI, Pattichis CS, Pantziaris MS, Nicolaides AN (2003) Texture-based classifi- 738 cation of atherosclerotic carotid plaques. IEEE Trans Med Imaging 22(7):902-912 739

31. Belcaro G, Nicolaides AN, Laurora G, Cesarone MR, De Sanctis M, Incandela L, Barsotti A 740 (1996) Ultrasound morphology classification of the arterial wall and cardiovascular events in a 741 6-year follow-up study. Arterioscl Thromb Vasc Biol 16:851-856 742

32. Schmidt C, Wendelhag I (1999) How can the variability in ultrasound measurements of intima- 743 media thickness be reduced? Studies of interobserver variability in carotid and femoral arteries. 744 $\begin{array}{ll}\text { Clinic Physiol 19(1):45-55 } & 745\end{array}$

33. Ellis SM, Sidhu PS (2000) Granularity of the carotid artery intima-medial layer: reproducibil- 746 ity of quantification by a computer-based program. Br J Radiol 37:595-600 


\section{Editor's Proof}

34. Bartolomucci F, Paterni M, Morizzo C, Kozakova M et al (2001) Early structural changes of 748 carotid artery in familial hypercholesterolemia. J Clin Hypertens 14:125A-126A $\quad 749$

35. Lind L, Andersson J, Roenn M, Gustavsson T (2007) The echogenecity of the intima-media 750 complex in the common carotid artery is closely related to the echogenecity in plaques. 751 Atherosclerosis 195:411-414

36. (2004) Prevention of disampling and fatal strokes by successful carotid endarterec- 753 tomy in patients without recent neurological symptoms: randomized control trial, Lancet 754 363(9420):1491-1502

37. Rosfors S, Hallerstam S, Jensen-Urstad K, Zetterling M, Carlstroem C (1998) Relationship 756 between intima-media thickness in the common carotid artery and atherosclerosis in the carotid 757 bifurcation. Stroke 29:1378-1382

38. Balasundaram JK, Wahida Banu RSD (2006) A non-invasive study of alterations of the carotid 759 artery with age using ultrasound images. Med Biol Eng Comput 44:67-72 760

39. Haralick RM, Shanmugam K, Dinstein I (1973) Texture features for image classification. IEEE 761 Trans Syst Man Cybern SMC 3:610-621

40. Weszka JS, Dyer CR, Rosenfield A (1976) A comparative study of texture measures for terrain 763 classification. IEEE Trans Syst Man Cybern SMC 6:269-285 764

41. Amadasun M, King R (1989) Textural features corresponding to textural properties. IEEE 765 Trans Syst Man Cybern 19(5):1264-1274

42. Wu CM, Chen YC, Hsieh K-S (1992) Texture features for classification of ultrasonic images. 767 IEEE Trans Med Imaging 11:141-152 768

43. Litwin M, Wuehl E, Jourdan C, Trelewicz J, Niemirska A, Fahr K, Jobs K, Grena R, Wawer 769 ZT, Rajszys P, Troeger J, Mehls O, Shaefer F (2005) Altered morphological properties of large 770 arteries in children with chronic renal failure and after and after renal transplantation. J Am 771 Soc Nephrol 16:1494-1500

44. Goncalves I,Lindholm MW, Pedro LM et al (2004) Elastin and calcium rather than collagen or 773 lipid content are associated with echogenecity of human carotid plaques. Stroke 35:795-800 774

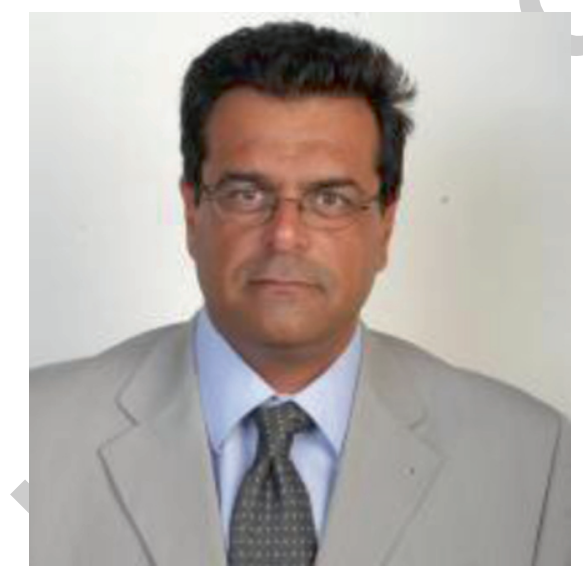

Christos P. Loizou was born in Cyprus 775 in October 1962 and received his BSc de- 776 gree in Electrical Engineering, the Dipl.- 777 Ing. (MSc) degree in Computer Sci- 778 ence and Telecommunications from the 779 University of Kaisserslautern, Kaisser- 780 slautern, Germany, and the PhD degree 781 from the Department of Computer Sci- 782 ence, Kingston University, London, UK, 783 on ultrasound image analysis of the 784 carotid artery in 1990 and 2005, respec- 785 tively. He is currently an Assistant Pro- 786 fessor with the Department of Computer 787 Science at Intercollege in Cyprus. His 788 research interests include medical imaging, in investigating the risk of stroke and 789 the multiple sclerosis disease. 


\section{Editor's Proof}

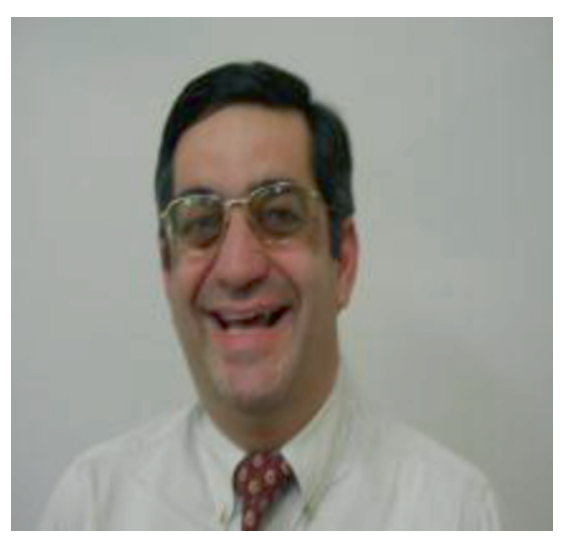

Constantinos S. Pattichis was born in 791 Cyprus on January 30, 1959, and received 792 his diploma as a technician engineer from 793 the Higher Technical Institute in Cyprus 794 in 1979, BSc in Electrical Engineering 795 from the University of New Brunswick, 796 Canada, in 1983, MSc in Biomedical En- 797 gineering from the University of Texas at 798 Austin, USA, in 1984, MSc in Neurology 799 from the University of Newcastle Upon 800 Tyne, UK, in 1991, and PhD in Elec- 801 tronic Engineering from the University 802 of London, UK, in 1992. He is currently 803 Professor with the Department of Computer Science of the University of Cyprus. 804 His research interests include ehealth, medical imaging, biosignal analysis, and 805 intelligent systems. 